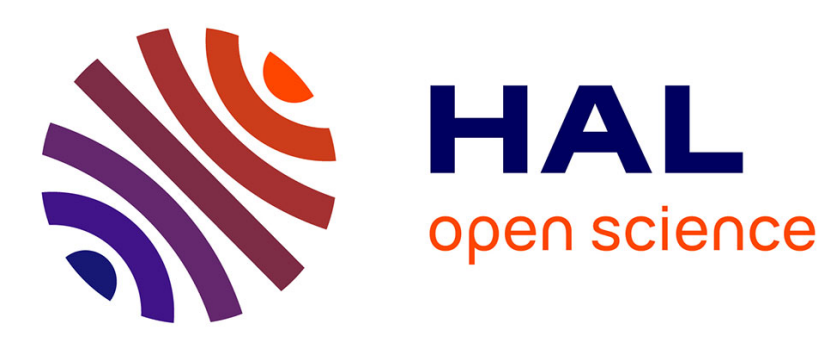

\title{
Equilibrium versions of set-valued variational principles and their applications to organizational behavior
} Jing-Hui Qiu, Antoine Soubeyran, Fei He

\section{To cite this version:}

Jing-Hui Qiu, Antoine Soubeyran, Fei He. Equilibrium versions of set-valued variational principles and their applications to organizational behavior. Optimization, 2020, 69 (12), pp.2657-2693. 10.1080/02331934.2020.1718127 . hal-02465110

\section{HAL Id: hal-02465110 https://hal-amu.archives-ouvertes.fr/hal-02465110}

Submitted on 19 Feb 2021

HAL is a multi-disciplinary open access archive for the deposit and dissemination of scientific research documents, whether they are published or not. The documents may come from teaching and research institutions in France or abroad, or from public or private research centers.
L'archive ouverte pluridisciplinaire HAL, est destinée au dépôt et à la diffusion de documents scientifiques de niveau recherche, publiés ou non, émanant des établissements d'enseignement et de recherche français ou étrangers, des laboratoires publics ou privés. 


\title{
Equilibrium versions of variational principles in quasi-metric spaces and the robust trap problem
}

\author{
Jing-Hui Qiu ${ }^{a}$, Fei He ${ }^{b}$ and Antoine Soubeyran ${ }^{c}$ \\ ${ }^{a}$ School of Mathematical Sciences, Soochow University, Suzhou, P.R. China; bSchool of Mathematical Sciences, Inner \\ Mongolia University, Hohhot, P.R. China; 'Aix-Marseille School of Economics, Aix-Marseille University, Marseille, \\ France
}

\begin{abstract}
Using a pre-order principle in [Qiu JH. A pre-order principle and set-valued Ekeland variational principle. J Math Anal Appl. 2014;419:904-937], we establish a general equilibrium version of setvalued Ekeland variational principle (denoted by EVP), where the objective bimap is defined on the product of left-complete quasi-metric spaces and taking values in a quasi-order linear space, and the perturbation consists of the quasi-metric and a positive vector $k_{0}$. Here, the ordering is only to be $k_{0}$-closed, which is strictly weaker than to be topologically closed. From the general equilibrium version, we deduce a number of particular equilibrium versions of EVP with set-valued bimaps or with vector-valued bimap. As applications of the equilibrium versions of EVP, we present several interesting results on equilibrium problems, vector optimization and fixed point theory in the setting of quasi-metric spaces. These results extend and improve the related known results. Using the obtained EVPs, we further study the existence and the robustness of traps in Behavioural Sciences.
\end{abstract}

\section{KEYWORDS}

Pre-order principle; equilibrium version of Ekeland variational principle; quasi-metric space; Caristi's fixed point theorem; robust trap problem

\section{Introduction}

As well-known, Ekeland variational principle (briefly, denoted by EVP) is a powerful tool in many subjects, such as non-linear analysis, applied mathematical analysis and optimization problems (see [1-4]). Motivated by its wide applications, many authors have been interested in extending EVP to the case with vector-valued or set-valued maps, see for example, [3-24] and the references therein. Oettli and Théra [25] started the study of the equilibrium versions of EVP. By an equilibrium problem we understand the problem of finding

$$
\bar{x} \in X \text { such that } f(\bar{x}, y) \geq 0 \text { for all } y \in X,
$$

where $X$ is a given set and $f: X \times X \rightarrow \mathbf{R}$ is a given function. This general problem was named 'equilibrium problem' by Blum and Oettli [26]. The equilibrium theory is a unified method for solving several problems, for example, optimization problem, saddle point problem, Nash equilibrium problem, variational inequality problem, complementarity problem, fixed point problem, minimax problem, etc. Recently, equilibrium versions of EVP have attracted a lot of attention and there have been many interesting results on this topic, see for example, [27-37]. In [30], Bianchi Kassay and Pini established an equilibrium version of EVP for quasi-lower semi-continuous vector-valued functions and obtained the existence result of solutions for vector equilibrium problems in compact 
and noncompact domains, respectively. Zeng and Li [37] further considered a set-valued equilibrium version of EVP and obtained existence results on solutions for set-valued vector equilibrium problems without any convexity assumptions. In order to deal with the case of set-valued maps, Gong [32] developed the scalarization method with positive linear functionals and provided a new equilibrium version of set-valued EVP which extends [30, Theorem 1] from vector-valued maps to set-valued maps. Moreover, he derived an existence theorem for solutions of set-valued vector equilibrium problems under a weaker condition, which improves [37, Theorem 4.1]. Using Gerstewitz's functions instead of positive linear functionals, Qiu [36] further developed Gong's method and established a new equilibrium version of EVP in the setting of metric spaces. Applying the new version, he derived some existence theorems on solutions of set-valued equilibrium problems, which improve the related results in [32,37]. We also remark that Alleche and Rădulescu [38], László and Viorel [39], and Kristály and Varga [40] investigated the set-valued equilibrium problems and obtained a number of interesting results on the existence of solutions and fixed point theory.

On the other hand, recently there have been several interesting results concerning completeness of quasi-metric spaces and EVPs in the setting of quasi-metric spaces (see for example, [41-48]). Here, a bifunction $q: X \times X \rightarrow \mathbf{R}_{+}:=[0,+\infty)$ on a nonempty set $X$ is said to be a quasi-metric on $X$ iff for all $x, y, z \in X$ it satisfies

(q1) $q(x, y) \geq 0$ (nonnegativity);

(q2) $q(x, y)=0 \Longleftrightarrow x=y$ (coincidence axiom);

(q3) $q(x, z) \leq q(x, y)+q(y, z)$ (triangle axiom).

If a quasi-metric $q$ enjoys the axiom of symmetry,

(q4) $q(x, y)=q(y, x)$,

then it is called a metric.

We adopt the notion from [47]. In [41-43], the quasi-metric space is called a $T_{1}$ quasi-metric space. Very recently, Bao, Khanh, Mordukhovich and Soubeyran [44-47] presented interesting applications of quasi-metric versions of EVP to psychology, behavioural sciences, capability theory of wellbeing and the modelization of organizational change. As pointed out in [47], in the variational rationality approach in $[49,50]$, costs-to-be-able-to-change verifies, in the simplest prototype case, the following four assumptions:

(1) no change no cost $C(x, x)=0, \forall x \in X$;

(2) it is not free to perform a move $C(x, y)>0, \forall x \neq y$;

(3) a direct change cost less than any indirect change $C(x, y) \leq C(x, z)+C(y, z), \forall x, y, z \in X$;

(4) the cost to perform a change cannot be equal to the cost to undo that change $C(x, y) \neq$ $C(y, x), \forall x, y \in X$.

Mathematically, such a cost function is indeed a quasi-metric on $X$. Hence, quasi-metric versions of EVP arouse our great interest.

In this paper, inspired by the above works, we consider equilibrium versions of set-valued and vector-valued EVPs in the setting of quasi-metric spaces and their applications. Using a pre-order principle in [20], we establish a general equilibrium version of set-valued EVP, where the objective bimap is defined on the product of left-complete quasi-metric spaces and taking values in a quasiorder linear space, and the perturbation consists of the quasi-metric and a positive vector. Here, the ordering cone needn't be topologically closed; it is only required to be $k_{0}$-closed, where $k_{0}$ is a positive vector. The objective bimap only needs to be sequential lower monotonicity with respect to the quasimetric, which is strictly weaker than the usual assumption on lower semi-continuity, and the lower boundedness of the objective bimap is taking the weakest form. From the general equilibrium version, we deduce a number of particular equilibrium versions of EVP with set-valued bimaps or with vectorvalued bimaps in the setting of quasi-metric spaces, which extend and improve the related results 
in $[30,32,37,41,45,47,48]$. Since a quasi-metric does not enjoy the axiom of symmetry, it needs to indicate left direction or right direction when one considers some notions on analysis and topology, such as neighbourhoods, limits, completeness and closedness. Though our main results are stated in left version, the corresponding results in right version still hold. As applications of the equilibrium versions of EVP, we present several interesting results in vector optimization, equilibrium problems and fixed point theory in the setting of quasi-metric spaces. These results extend and improve the related results in $[25,27,29-32,37,41,43,47]$.

In the final part of the paper, we consider the applications of our set-valued equilibrium EVPs to behavioural sciences, especially to studying the robust trap problem. This paper is motivated by applications. It has two sides. At the mathematical level, this paper offers, as seen before, a general equilibrium version of the set valued Ekeland variational principle (Ekeland $[1,2]$ ). At the behavioural level, this paper considers the modelization and the resolution of the important trap problem in Behavioural Sciences. In his (VR) variational rationality approach of the dynamics of human behaviours Soubeyran [49,50] showed, among a lot of applications, how the Ekeland variational principle (Ekeland $[1,2]$ ) and its equivalent formulations give sufficient conditions for the existence of traps. This paper adds the consideration of an important aspect, the robustness of traps, that is, how they resist to exogenous variations of the environment and the internal state of the agent, or group of agents. In this way, its enrichs the resolution of the trap problem in Behavioural Sciences and differs from a lot of generalizations of variational principles in Mathematics which come with no computational or real life justifications.

The rest of the paper is organized as follows. Section 2 presents preliminary results on convex cones in real linear spaces and on quasi-metric spaces, which are useful in deriving our main results. In Section 3, we first recall a pre-order principle in [20]. Using the pre-order principle, we deduce several equilibrium versions of set-valued and vector-valued EVPs in quasi-metric spaces. In Section 4, we apply equilibrium versions of EVPs in quasi-metric spaces to equilibrium problems, vector optimization and fixed point theorems. In Section 5, using the obtained EVPs, we study the robust trap problem in behavioural sciences.

\section{Preliminary results on convex cones in real linear spaces and on quasi-metric spaces}

A useful approach for solving a vector problem is to reduce it to a scalar problem. Gerstewitz's functions introduced in [51] are often used as the basis of the scalarization. In the framework of topological vector spaces (briefly, denoted by t.v.s.), Gerstewitz's functions generated by closed convex (solid) cones and their properties have been investigated thoroughly, for example, see $[3,4,21,22,52]$ and the references therein. In order to give more general version of set-valued (or vector-valued) EVP, we need to consider Gerstewitz's functions generated by general convex cones in real linear spaces and re-examine their properties in the more general setting. The main ideas originate from $[3,4,52]$.

In the following, we always assume that $Y$ is a real linear space. If $A, B \subset Y$ and $\alpha \in \mathbf{R}$, the sets $A+B$ and $\alpha A$ are defined as follows:

$$
\begin{aligned}
A+B & :=\{z \in Y: \exists x \in A, \exists y \in B \text { such that } z=x+y\} \\
\alpha A & :=\{z \in Y: \exists x \in A \text { such that } z=\alpha x\} .
\end{aligned}
$$

For a nonempty subset $A \subset Y$, the vector closure of $A$ is defined as follows (refer to [53]):

$$
\operatorname{vcl}(A)=\left\{y \in Y: \exists v \in Y, \exists \lambda_{n} \geq 0, \lambda_{n} \rightarrow 0 \text { such that } y+\lambda_{n} v \in A, \forall n \in N\right\} .
$$


For any given $v_{0} \in Y$, we define the $v_{0}$-vector closure (briefly, $v_{0}$-closure) of $A$ as follows:

$$
\operatorname{vcl}_{v_{0}}(A)=\left\{y \in Y: \exists \lambda_{n} \geq 0, \lambda_{n} \rightarrow 0 \text { such that } y+\lambda_{n} v_{0} \in A, \forall n \in N\right\} .
$$

Obviously,

$$
A \subset \operatorname{vcl}_{v_{0}}(A) \subset \cup_{v \in Y} \operatorname{vcl}_{v}(A)=\operatorname{vcl}(A) .
$$

All the above inclusions are proper. Moreover, if $Y$ is a topological vector space (briefly, denoted by t.v.s.) and $\operatorname{cl}(A)$ denotes the closure of $A$, then $\operatorname{vcl}(A) \subset \operatorname{cl}(A)$ and the inclusion is also proper. $\mathrm{A}$ subset $A$ of $Y$ is said to be topologically closed if $A=\operatorname{cl}(A)$; to be vectorially closed if $A=\operatorname{vcl}(A)$; to be $v_{0}$-closed if $A=\operatorname{vcl}_{v_{0}}(A)$. In general, a nonempty set $A \subset Y$ need not be $v_{0}$-closed,; a $v_{0}$-closed set need not be vectorially closed and a vectorially closed set need not be topologically closed (for details, see [21]). A nonempty set $K \subset Y$ is called a convex cone if $K+K \subset K$ and $\alpha K \subset K$ for any $\alpha \geq 0$. A convex cone $K$ can specify a quasi-order $\leq_{K}$ (i.e. a binary relation satisfying reflexive and transitive properties) on $Y$ as follows:

$$
y_{1}, y_{2} \in Y, \quad y_{1} \leq_{K} y_{2} \Longleftrightarrow y_{1}-y_{2} \in-K
$$

In this case, $K$ is called the ordering cone or positive cone. And every $k \in K \backslash-K$ is called a positive vector. We always assume that $K$ is nontrivial, i.e. $K \neq\{0\}$ and $K \neq Y$. We can easily prove the following:

Proposition 2.1 (see [21]): Let $k_{0} \in K \backslash-K$. Then,

(i) $\operatorname{vcl}_{k_{0}}(K)$ is a convex cone containing $K \cup\{0\}$, but not containing $-k_{0}$;

(ii) for any sequence $\left\{\epsilon_{n}\right\}$ with $\epsilon_{n}>0$ and $\epsilon_{n} \rightarrow 0$,

$$
\operatorname{vcl}_{k_{0}}(K)=\cap_{\epsilon>0}\left(K-\epsilon k_{0}\right)=\cap_{n=1}^{\infty}\left(K-\epsilon_{n} k_{0}\right) ;
$$

For any $y \in Y$, if there exists $t \in \mathbf{R}$ such that $y \in t k_{0}-K$, then for any $t^{\prime}>t, y \in t^{\prime} k_{0}-K$. Thus, we can define a function $\xi_{k_{0}}: Y \rightarrow \mathbf{R} \cup\{ \pm \infty\}$ as follows: if there exists $t \in \mathbf{R}$ such that $y \in t k_{0}-K$, then define $\xi_{k_{0}}(y)=\inf \left\{t \in \mathbf{R}: y \in t k_{0}-K\right\}$; or else, define $\xi_{k_{0}}(y)=+\infty$. Such a function is called a Gerstewitz's function generated by $K$ and $k_{0}$.

Proposition 2.2 (see [21, Lemma 2.6]): There exists $z \in Y$ such that $\xi_{k_{0}}(z)=-\infty$ iff $k_{0} \in-\operatorname{vcl}(K)$.

Proposition 2.3 (see $[3,4,21,22]$ ): Let $K \subset Y$ be a convex cone and $k_{0} \in K \backslash-\operatorname{vcl}(K)$. Then, the Gerstewitz's function $\xi_{k_{0}}$ has the following properties:

(i) $y_{1} \leq_{K} y_{2} \Longrightarrow \xi_{k_{0}}\left(y_{1}\right) \leq \xi_{k_{0}}\left(y_{2}\right), \forall y_{1}, y_{2} \in Y$;

(ii) $\xi_{k_{0}}(\alpha y)=\alpha \xi_{k_{0}}(y), \forall y \in Y, \forall \alpha \geq 0$;

(iii) $\xi_{k_{0}}\left(y_{1}+y_{2}\right) \leq \xi_{k_{0}}\left(y_{1}\right)+\xi_{k_{0}}\left(y_{2}\right), \forall y_{1}, y_{2} \in Y$;

Let $y \in Y$ and $r \in \mathbf{R}$. Then, we have:

(iv) $\xi_{k_{0}}(y)<r \Longleftrightarrow y \in r k_{0}-\operatorname{vint}_{k_{0}}(K)$, where $\operatorname{vint}_{k_{0}}(K)=(0,+\infty) k_{0}+K$;

(v) $\xi_{k_{0}}(y) \leq r \Longleftrightarrow y \in r k_{0}-\operatorname{vcl}_{k_{0}}(K)$;

(vi) $\xi_{k_{0}}(y)=r \Longleftrightarrow y \in r k_{0}-\left(\operatorname{vcl}_{k_{0}}(K) \backslash \operatorname{vint}_{k_{0}}(K)\right)$;

Particularly, $\xi_{k_{0}}(0)=0, \quad \xi_{k_{0}}\left(k_{0}\right)=1$;

(vii) $\xi_{k_{0}}(y) \geq r \Longleftrightarrow y \notin r k_{0}-\operatorname{vint}_{k_{0}}(K)$;

(viii) $\xi_{k_{0}}(y)>r \Longleftrightarrow y \notin r k_{0}-\operatorname{vcl}_{k_{0}}(K)$;

(ix) $\xi_{k_{0}}\left(y+r k_{0}\right)=\xi_{k_{0}}(y)+r$.

Next, we turn to some notions related to quasi-metric spaces; please refer to [41-48]. 
Definition 1: Let $(X, q)$ be a quasi-metric space and $\left\{x_{n}\right\}$ be a sequence in $X$.

The sequence $\left\{x_{n}\right\}$ is said to be left-convergent to a point $\bar{x} \in X$ iff $q\left(x_{n}, \bar{x}\right) \rightarrow 0(n \rightarrow \infty)$.

The sequence $\left\{x_{n}\right\}$ is said to be left-Cauchy iff for every $\epsilon>0$ there exists $n_{\epsilon} \in \mathbf{N}$ such that

$$
q\left(x_{n}, x_{m}\right)<\epsilon, \forall m \geq n \geq n_{\epsilon} .
$$

The quasi-metric space $(X, q)$ is said to be left-complete iff every left-Cauchy sequence is leftconvergent.

A nonempty subset $S \subset X$ is said to be left-closed iff a sequence $\left\{x_{n}\right\} \subset S$ and $q\left(x_{n}, \bar{x}\right) \rightarrow 0(n \rightarrow$ $\infty)$ imply that $\bar{x} \in S$.

The quasi-metric space $(X, q)$ is said to be left-Hausdorff iff every left-convergent sequence has the unique limit, that is, if there exists $\bar{x}, \bar{y} \in X$ such that $q\left(x_{n}, \bar{x}\right) \rightarrow 0(n \rightarrow \infty)$ and $q\left(x_{n}, \bar{y}\right) \rightarrow$ $0(n \rightarrow \infty)$, then one has $\bar{x}=\bar{y}$.

Since a quasi-metric does not enjoy the axiom of symmetry, one can also define the corresponding notions, such as right-convergence, right-Cauchy, right-completeness, right-closed and rightHausdorff. As we know, a left-convergent sequence is not necessarily left-Cauchy and a quasi-metric space might not be left-Hausdorff, for example, see [44,47] and the references therein. Consider $X=[0,1]$ and the quasi-metric $q$ on $X$ defined by

$$
q(x, y)=\left\{\begin{array}{cc}
x-y, & \text { if } x \geq y \\
1+x-y, & \text { if } x<y \text { but }(x, y) \neq(0,1) \\
1, & \text { if }(x, y)=(0,1)
\end{array}\right.
$$

It is easy to check that the quasi-metric space $(X, q)$ is not left-Hausdorff since the sequence $\left\{x_{n}\right\}$ with $x_{n}=1 / n$ satisfies $q\left(x_{n}, \bar{x}\right) \rightarrow 0(n \rightarrow \infty)$ and $q\left(x_{n}, \bar{y}\right) \rightarrow 0(n \rightarrow \infty)$, where $\bar{x}=0$ and $\bar{y}=1$.

Now, we consider topologies generated by quasi-metrics. Let $(X, q)$ be a quasi-metric space, $x_{0} \in X$ and $\epsilon>0$ be given. We denote the set $\left\{x \in X: q\left(x, x_{0}\right)<\epsilon\right\}$ by $B_{l}\left(x_{0}, \epsilon\right)$, which is called the left-open ball of radius $\epsilon$ centred at $x_{0}$. A set $U$ is called a left-neighbourhood of $x_{0}$ iff there exists $\epsilon>0$ such that $U \supset B_{l}\left(x_{0}, \epsilon\right)$. We usually denote it by $U_{l}\left(x_{0}\right)$. The left-topology $\tau_{l}(q)$ of a quasi-metric space $(X, q)$ can be defined starting from the family $\left\{U_{l}(x)\right\}$ of neighbourhoods of an arbitrary point $x \in X$. A set $G \subset X$ is $\tau_{l}(q)$-open iff for every $x \in G$ there exists $\epsilon=\epsilon_{x}>0$ such that $B_{l}(x, \epsilon) \subset G$. Obviously, the convergence of a sequence $\left\{x_{n}\right\}$ to $x$ with respect to $\tau_{l}(q)$ is exactly the left-convergence, i.e. $q\left(x_{n}, x\right) \rightarrow 0(n \rightarrow \infty)$. Similarly, we can define right-open balls, right-neighbourhoods, the righttopology, $\tau_{r}(q), \tau_{r}(q)$-open sets, right-convergence, right-completeness and right-closed sets. Since (q2) holds, both $\tau_{l}(q)$ and $\tau_{r}(q)$ are $T_{1}$-topology (see [41,42]). As a space with two topologies, $\tau_{l}(q)$ and $\tau_{r}(q)$, a quasi-metric space can be viewed as a bitopological space in the sense of Kelly (see [54]). Remark that the left-topology $\tau_{l}(q)$ is exactly the topology $\tau(\bar{q})$, where $\bar{q}(x, y)=q(y, x), \forall x, y \in X$, and the right-topology $\tau_{r}(q)$ is exactly the topology $\tau(q)$ in $[41,42]$.

As we have seen above, a quasi-metric space might not be left-Hausdorff. Then, a question arises: under which conditions does a quasi-metric space enjoy the uniqueness of left-limits? In fact, the uniqueness of left-limits is equivalent to that the left-topology generated by the quasi-metric is Hausdorff. In the following, we give a condition which can ensure that the left-limit is unique. The condition is as follows: there exists a function $\varphi:(0,+\infty) \rightarrow(0,+\infty)$ satisfying $\varphi(t) \rightarrow 0$ when $t \rightarrow 0$ such that for any $x, y \in X, q(x, y) \leq \varphi(q(y, x))$. Let a sequence $\left(x_{n}\right) \subset X$ such that

$$
q\left(x_{n}, \bar{x}\right) \rightarrow 0(n \rightarrow \infty) \text { and } q\left(x_{n}, \bar{y}\right) \rightarrow 0(n \rightarrow \infty) .
$$

Then,

$$
\begin{aligned}
q(\bar{x}, \bar{y}) & \leq q\left(\bar{x}, x_{n}\right)+q\left(x_{n}, \bar{y}\right) \\
& \leq \varphi\left(q\left(x_{n}, \bar{x}\right)\right)+q\left(x_{n}, \bar{y}\right) \rightarrow 0(n \rightarrow \infty) .
\end{aligned}
$$


Thus, $q(\bar{x}, \bar{y})=0$ and $\bar{x}=\bar{y}$. This means that the left limit is unique. Particularly, the function $\varphi$ may be taken as $\varphi(t)=a t$, where $a>0$ is a constant.

At the behavioural level (see Section 5), the uniqueness of the left-limit works when costs of being able to change $C(x, y)$ are not too as symmetric, that is when $C(x, y) \leq a C(y, x), a>0$, for all $x, y \in X$. This is a very reasonable assumption at the behavioural level ( $a$ can be very large).

Then, left convergence $C\left(x_{n}, \bar{x}\right) \rightarrow 0, n \rightarrow+\infty$ and $C\left(x_{n}, \bar{y}\right) \rightarrow 0, n \rightarrow+\infty$ imply $0 \leq$ $C(\bar{x}, \bar{y}) \leq C\left(\bar{x}, x_{n}\right)+C\left(x_{n}, \bar{y}\right) \leq a C\left(x_{n}, \bar{x}\right)+C\left(x_{n}, \bar{y}\right)$. Because, by hypothesis, the right hand side of this last inequality goes to zero, the result follows.

As we know, in the original version of Ekeland's principle, the objective function $f$ is required to be lower semi-continuous. Later, the assumption of lower semi-continuity has been weakened as the sequential lower monotonicity. At the end of this section, we consider the semi-continuity and the sequential lower monotonicity of set-valued maps defined on quasi-metric spaces. For the definiteness, we only relate the notions in 'left' direction and the corresponding notions in 'right' direction can be similarly defined. In the following, we always assume that $(X, q)$ is a quasi-metric space and $Y$ is a t.v.s. with the quasi-order $\leq_{K}$ induced by a convex cone $K$.

Definition 2 (refer to [12,14,32,37]): A set-valued map $f: X \rightarrow 2^{Y} \backslash\{\emptyset\}$ is said to be left- $K$-upper semi-continuous at $x_{0} \in X$, iff for any neighbourhood $V$ of 0 in $Y$, there exists a left-neighbourhood $U_{l}\left(x_{0}\right)$ of $x_{0}$ such that

$$
f(x) \subset f\left(x_{0}\right)+V-K, \quad \forall x \in U_{l}\left(x_{0}\right) .
$$

And $f$ is said to be left- $K$-upper semi-continuous on $X$ iff it is left - $K$-upper semi-continuous at every $x \in X$.

A set-valued map $f: X \rightarrow 2^{Y} \backslash\{\emptyset\}$ is said to be left- $K$-lower semi-continuous at $x_{0} \in X$, iff for any $y_{0} \in f\left(x_{0}\right)$ and for any neighbourhood $V$ of 0 in $Y$, there exists a left-neighbourhood $U_{l}\left(x_{0}\right)$ of $x_{0}$ such that

$$
f(x) \cap\left(y_{0}+V+K\right) \neq \emptyset, \quad \forall x \in U_{l}\left(x_{0}\right) .
$$

And $f$ is said to be left- $K$-lower semi-continuous on $X$ iff it is left- $K$-lower semi-continuous at every $x \in X$.

Remark 1: If $f: X \rightarrow Y$ is a vector-valued map, then by Definition 2, we have the following results:

$f$ is left- $K$-upper semi-continuous at $x_{0} \in X$, iff for any neighbourhood $V$ of 0 in $Y$, there exists a left-neighbourhood $U_{l}\left(x_{0}\right)$ of $x_{0}$ such that

$$
f(x) \in f\left(x_{0}\right)+V-K, \quad \forall x \in U_{l}\left(x_{0}\right) .
$$

$f$ is left- $K$-lower semi-continuous at $x_{0} \in X$, iff for any neighbourhood $V$ of 0 in $Y$, there exists a left-neighbourhood $U_{l}\left(x_{0}\right)$ of $x_{0}$ such that

$$
f(x) \in f\left(x_{0}\right)+V+K, \quad \forall x \in U_{l}\left(x_{0}\right) .
$$

Since $V$ may be taken a circled set (see [55]), i.e. $V$ consists of all $\alpha x, x \in V,|\alpha| \leq 1$, then by (2.1) and (2.2), we know that $f$ is left- $K$-upper semi-continuous at $x_{0} \in X$ iff $-f$ is left- $K$-lower semi-continuous at $x_{0} \in X$.

Definition 3: A set-valued map $f: X \rightarrow 2^{Y} \backslash\{\emptyset\}$ is said to be quasi left- $K$-lower semi-continuous at $x_{0} \in X$, iff for any $b \in Y$ with $f\left(x_{0}\right) \not \subset b-K$, there exists a left-neighbourhood $U_{l}\left(x_{0}\right)$ of $x_{0}$ such that

$$
f(x) \not \subset b-K, \quad \forall x \in U_{l}\left(x_{0}\right) .
$$

And $f$ is said to be quasi left- $K$-lower semi-continuous on $X$ iff $f$ is quasi left- $K$-lower semi-continuous at every $x \in X$.

It is easy to prove the following two propositions. Their proofs are omitted 
Proposition 2.4: A set-valued map $f: X \rightarrow 2^{Y} \backslash\{\emptyset\}$ is quasi left-K-lower semi-continuous on $X$ iff for each $b \in Y$, the set $\{x \in X: f(x) \subset b-K\}$ is left-closed.

Proposition 2.5: $\quad$ Moreover, assume that $K$ is closed. Then, $f: X \rightarrow 2^{Y} \backslash\{\emptyset\}$ being left-K-lower semi-continuous on $X$ implies that $f$ is quasi left-K-lower semi-continuous on $X$.

As we know, when the domain $X$ is endowed with the metric $d$, then a vector-valued map $f$ : $X \rightarrow\left(Y, \leq_{K}\right)$ is said to be $K$-sequentially lower monotone (briefly, denoted by $K$-slm or slm) iff $f\left(x_{n+1}\right) \leq_{K} f\left(x_{n}\right), \forall n$, and $x_{n} \rightarrow \bar{x}$ imply that $f(\bar{x}) \leq_{K} f\left(x_{n}\right), \forall n$ (see [13]). In [10], slm is called (H4); in [6], slm is called monotonical semi-continuity; and in [34], slm is called lower semi-continuity from above (briefly, lsca). In general, for vector-valued maps, we have: lower semi-continuity $\Rightarrow$ quasi lower semi-continuity $\Rightarrow \operatorname{slm}$. And none of the above converses is true. For details, see $[8,16]$. Now, for maps defined on quasi-metric spaces, we give the following definition.

Definition 4: Let $(X, q)$ be a quasi-metric space and $Y$ be a real linear space with the quasi-order $\leq_{K}$ induced by a convex cone $K \subset Y$. A vector-valued map $f: X \rightarrow Y$ is said to be left- $K$ sequentially lower monotone (briefly, denoted by left- $K$-slm or left-slm) iff $f\left(x_{n+1}\right) \leq_{K} f\left(x_{n}\right), \forall n$, and $q\left(x_{n}, \bar{x}\right) \rightarrow 0$ imply that $f(\bar{x}) \leq_{K} f\left(x_{n}\right), \forall n$.

For extended real-valued functions, Bao, Khanh and Soubeyran (see [47]) have already given the definition of left- $K$-slm. They call a function $f:(X, q) \rightarrow \mathbf{R} \cup\{+\infty\}$ to be decreasing left-lowersemicontinuous iff $f\left(x_{n+1}\right) \leq f\left(x_{n}\right), \forall n$, and $q\left(x_{n}, \bar{x}\right) \rightarrow 0$ imply that $f(\bar{x}) \leq f\left(x_{n}\right), \forall n$. Furthermore, Qiu [35] extended slm to bimaps. Now, for vector-valued bimaps and set-valued bimaps defined on quasi-metric spaces, we can also give the definition of left- $K$-slm as follows:

Definition 5: Let $(X, q)$ and $Y$ be the same as in Definition 4. A vector-valued bimap $F: X \times X \rightarrow$ $\left(Y, \leq_{K}\right)$ is said to be left- $K$-sequentially lower monotone (briefly, denoted by left- $K$-slm or leftslm) iff $F\left(x_{n}, x_{n+1}\right) \leq_{K} 0, \forall n$, and $q\left(x_{n}, \bar{x}\right) \rightarrow 0$ imply that $F\left(x_{n}, \bar{x}\right) \leq_{K} 0$. A set-valued bimap $F: X \times X \rightarrow 2^{Y} \backslash\{\emptyset\}$ is said to be left- $K$-sequentially lower monotone (briefly, denoted by left- $K$ slm or left-slm) iff $F\left(x_{n}, x_{n+1}\right) \subset-K, \forall n$, and $q\left(x_{n}, \bar{x}\right) \rightarrow 0$ imply that $F\left(x_{n}, \bar{x}\right) \subset-K, \forall n$.

Proposition 2.6: Let a set-valued bimap $F: X \times X \rightarrow 2^{Y} \backslash\{\emptyset\}$ satisfy subadditivity, i.e. $F(x, z) \subset$ $F(x, y)+F(y, z)-K, \forall x, y, z \in X$. If for each $x \in X$, the map $y \mapsto F(x, y)$ is quasi left- $K$-lower semi-continuous, then $F$ is left-K-slm.

Proof: Let a sequence $\left\{x_{n}\right\} \subset X$ such that $F\left(x_{n}, x_{n+1}\right) \subset-K, \forall n$, and $q\left(x_{n}, \bar{x}\right) \rightarrow 0$. For any $p \in \mathbf{N}$,

$$
\begin{aligned}
F\left(x_{n}, x_{n+p}\right) & \subset F\left(x_{n}, x_{n+1}\right)+F\left(x_{n+1}, x_{n+p}\right)-K \\
& \subset \cdots \cdots \\
& \subset F\left(x_{n}, x_{n+1}\right)+F\left(x_{n+1}, x_{n+2}\right)+\cdots+F\left(x_{n+p-1}, x_{n+p}\right)-K \\
& \subset-K-K-\cdots-K=-K .
\end{aligned}
$$

From this,

$$
x_{n+p} \in\left\{y \in X: F\left(x_{n}, y\right) \subset-K\right\}, \forall p \in \mathbf{N} .
$$

By the assumption, for each $x \in X$, the map: $y \mapsto F(x, y)$ is quasi left- $K$-lower semi-continuous. By Proposition 2.4, for each $b \in Y$, the set $\{y \in X: F(x, y) \subset b-K\}$ is left-closed. Particularly, for each $n$, the set $\left\{y \in X: F\left(x_{n}, y\right) \subset-K\right\}$ is left-closed. Now, $\left\{x_{n+p}\right\}_{p \in \mathbf{N}} \subset\left\{y \in X: F\left(x_{n}, y\right) \subset-K\right\}$ and $q\left(x_{n+p}, \bar{x}\right) \rightarrow 0(p \rightarrow \infty)$, so $\bar{x} \in\left\{y \in X: F\left(x_{n}, y\right) \subset-K\right\}$, that is, $F\left(x_{n}, \bar{x}\right) \subset-K$. Thus, we have shown that $F$ is left- $K$-slm. 


\section{Equilibrium versions of set-valued and vector-valued EVPs in quasi-metric spaces}

For brevity, we call a function $\psi: X \rightarrow(0,+\infty)$ to be $F$-decreasing iff $\psi\left(x^{\prime}\right) \geq \psi(x)$ if $F\left(x, x^{\prime}\right) \subset$ $-K$, where $F: X \times X \rightarrow 2^{Y} \backslash\{\emptyset\}$ is a set-valued bimap. And we denote the set consisting of all such functions by $\Psi_{F}$.

Now we come to our main results.

Theorem 3.1: Let $(X, q)$ be a left-complete quasi-metric space, $Y$ be a real linear space, $K \subset Y$ be a convex cone and $k_{0} \in K \backslash-\operatorname{vcl}(K)$ such that $K$ is $k_{0}$-closed. Let $F: X \times X \rightarrow 2^{Y} \backslash\{\emptyset\}$ be a set-valued bimap satisfying the following conditions:

(i) $\exists x_{0} \in X$ such that $F\left(x_{0}, x_{0}\right) \subset-K$;

(ii) $\exists \alpha \in \mathbf{R}, \exists s_{0} \in(-\infty,+\infty) k_{0}-K$ such that

$$
F\left(x_{0}, X\right) \cap\left(-s_{0}+\alpha k_{0}-K\right)=\emptyset ;
$$

(iii) $F(x, z) \subset F(x, y)+F(y, z)-K, \forall x, y, z \in X$;

(iv) $F$ is left $K$-slm in $(X, q)$.

Assume that $\psi \in \Psi_{F}$.

Then, There exists $\bar{x} \in X$ such that

(a) $F\left(x_{0}, \bar{x}\right)+\psi\left(x_{0}\right) q\left(x_{0}, \bar{x}\right) k_{0} \subset-K$;

(b) $\forall x \in X \backslash\{\bar{x}\}, F(\bar{x}, x)+\psi(\bar{x}) q(\bar{x}, x) k_{0} \not \subset-K$.

In order to give the proof of Theorem 3.1, we need a pre-order principle proposed by Qiu (see [20]). As in [9], a binary relation $\preceq$ on $X$ is called a pre-order if it satisfies the transitive property. Let $(X, \preceq)$ be a pre-order set. An extended real-valued function $\eta:(X, \preceq) \rightarrow \mathbf{R} \cup\{ \pm \infty\}$ is called monotone with respect to $\preceq$ iff for any $x_{1}, x_{2} \in X$,

$$
x_{1} \preceq x_{2} \Longrightarrow \eta\left(x_{1}\right) \leq \eta\left(x_{2}\right) .
$$

For any given $x_{0} \in X$, denote $S\left(x_{0}\right)$ the set $\left\{x \in X: x \preceq x_{0}\right\}$. First, we recall a pre-order principle as follows.

Theorem 3.A: (see [20, Theorem 2.1]): Let $(X, \preceq)$ be a pre-order set, $x_{0} \in X$ such that $S\left(x_{0}\right) \neq \varnothing$ and $\eta:(X, \preceq) \rightarrow \mathbf{R} \cup\{ \pm \infty\}$ be an extended real-valued function which is monotone with respect to $\preceq$ Suppose that the following conditions are satisfied:

(A) $-\infty<\inf \left\{\eta(x): x \in S\left(x_{0}\right)\right\}<+\infty$;

(B) for any $x \in S\left(x_{0}\right)$ with $-\infty<\eta(x)<+\infty$ and $x^{\prime} \in S(x) \backslash\{x\}$, one has $\eta(x)>\eta\left(x^{\prime}\right)$;

(C) for any sequence $\left\{x_{n}\right\} \subset S\left(x_{0}\right)$ with $x_{n} \in S\left(x_{n-1}\right) \forall n$, such that $\eta\left(x_{n}\right)-\inf \{\eta(x): x \in$ $\left.S\left(x_{n-1}\right)\right\} \rightarrow 0(n \rightarrow \infty)$, there exists $u \in X$ such that $u \in S\left(x_{n}\right) \forall n$.

Then, there exists $\bar{x} \in X$ such that

(a) $\bar{x} \in S\left(x_{0}\right)$;

(b) $S(\bar{x}) \subset\{\bar{x}\}$.

Proof of Theorem 3.1: We shall apply Theorem 3.A to prove the result. Define a relation $\preceq$ on $X$ as follows: for any $x, x^{\prime} \in X$,

$$
x^{\prime} \preceq x \Longleftrightarrow F\left(x, x^{\prime}\right)+\psi(x) q\left(x, x^{\prime}\right) k_{0} \subset-K .
$$


It is easy to verify that $\preceq$ satisfies the transitive property. Hence $\preceq$ is a pre-order. For any $x \in X$, put $S(x):=\left\{x^{\prime} \in X: x^{\prime} \preceq x\right\}$. We define a function $\eta:(X, \preceq) \rightarrow \mathbf{R} \cup\{+\infty\}$ as follows:

$$
\eta(x):=\sup \left\{\xi_{k_{0}}\left(y+s_{0}\right): y \in F\left(x_{0}, x\right)\right\}, \quad x \in X
$$

First, we show that $\eta$ is monotone with respect to $\preceq$. Let $x, x^{\prime} \in X$ such that $x^{\prime} \preceq x$, i.e. $F\left(x, x^{\prime}\right)+$ $\psi(x) q\left(x, x^{\prime}\right) k_{0} \subset-K$. Thus,

$$
F\left(x, x^{\prime}\right) \subset-\psi(x) q\left(x, x^{\prime}\right) k_{0}-K .
$$

Combining this with condition (iii), we have

$$
\begin{aligned}
F\left(x_{0}, x^{\prime}\right) & \subset F\left(x_{0}, x\right)+F\left(x, x^{\prime}\right)-K \\
& \subset F\left(x_{0}, x\right)-\psi(x) q\left(x, x^{\prime}\right) k_{0}-K .
\end{aligned}
$$

Take any $y^{\prime} \in F\left(x_{0}, x^{\prime}\right)$, there exists $y \in F\left(x_{0}, x\right)$ such that

$$
y^{\prime} \in y-\psi(x) q\left(x, x^{\prime}\right) k_{0}-K
$$

Hence,

$$
y^{\prime}+s_{0} \in y+s_{0}-\psi(x) q\left(x, x^{\prime}\right) k_{0}-K .
$$

Acting on two sides of the above relation by $\xi_{k_{0}}$ and using Proposition 2.3, we have

$$
\begin{aligned}
\xi_{k_{0}}\left(y^{\prime}+s_{0}\right) & \leq \xi_{k_{0}}\left(y+s_{0}\right)-\psi(x) q\left(x, x^{\prime}\right) \\
& \leq \sup \left\{\xi_{k_{0}}\left(z+s_{0}\right): z \in F\left(x_{0}, x\right)\right\}-\psi(x) q\left(x, x^{\prime}\right) \\
& =\eta(x)-\psi(x) q\left(x, x^{\prime}\right) .
\end{aligned}
$$

From this, we have

$$
\eta\left(x^{\prime}\right) \leq \eta(x)-\psi(x) q\left(x, x^{\prime}\right) \leq \eta(x) .
$$

That is, $\eta$ is monotone with respect to $\preceq$. Particularly, if $x^{\prime} \preceq x$ and $x^{\prime} \neq x$ and $\eta(x)<+\infty$, then we have

$$
\eta\left(x^{\prime}\right) \leq \eta(x)-\psi(x) q\left(x, x^{\prime}\right)<\eta(x) .
$$

By condition (i), we easily verify that $x_{0} \in S\left(x_{0}\right)$ and $S\left(x_{0}\right) \neq \emptyset$. It is sufficient to prove that conditions (A), (B) and (C) in Theorem 3.A are satisfied.

Prove that (A) is satisfied. By condition (ii), $s_{0} \in(-\infty,+\infty) k_{0}-K$. Hence $\xi_{k_{0}}\left(s_{0}\right)<+\infty$. By condition (i), for any $y \in F\left(x_{0}, x_{0}\right), y \in-K$. Hence,

$$
\xi_{k_{0}}\left(y+s_{0}\right) \leq \xi_{k_{0}}\left(s_{0}\right)<+\infty
$$

Thus,

$$
\eta\left(x_{0}\right)=\sup \left\{\xi_{k_{0}}\left(y+s_{0}\right): y \in F\left(x_{0}, x_{0}\right)\right\} \leq \xi_{k_{0}}\left(s_{0}\right)<+\infty .
$$

By condition (ii), for any $x \in X$ and any $y \in F\left(x_{0}, x\right), y \notin-s_{0}+\alpha k_{0}-K$, so $\xi_{k_{0}}\left(y+s_{0}\right) \geq \alpha$ and

$$
\eta(x)=\sup \left\{\xi_{k_{0}}\left(y+s_{0}\right): y \in F\left(x_{0}, x\right)\right\} \geq \alpha .
$$

Thus,

$$
-\infty<\alpha \leq \inf \left\{\eta(x): x \in S\left(x_{0}\right)\right\} \leq \eta\left(x_{0}\right) \leq \xi_{k_{0}}\left(s_{0}\right)<+\infty .
$$

That is, $(\mathrm{A})$ is satisfied. 
Prove that (B) is satisfied. Let $x \in S\left(x_{0}\right)$ such that $-\infty<\eta(x)<+\infty$ and $x^{\prime} \in S(x) \backslash\{x\}$. By (3.1), we have $\eta\left(x^{\prime}\right)<\eta(x)$ and (B) is satisfied.

Prove that $(\mathrm{C})$ is satisfied. Let a sequence $\left\{x_{n}\right\} \subset S\left(x_{0}\right)$ such that $x_{n} \in S\left(x_{n-1}\right) \forall n$. Since $x_{1} \in S\left(x_{0}\right), x_{2} \in S\left(x_{1}\right), \cdots, x_{n} \in S\left(x_{n-1}\right)$, we have

$$
\begin{aligned}
& F\left(x_{0}, x_{1}\right)+\psi\left(x_{0}\right) q\left(x_{0}, x_{1}\right) k_{0} \subset-K, \\
& F\left(x_{1}, x_{2}\right)+\psi\left(x_{1}\right) q\left(x_{1}, x_{2}\right) k_{0} \subset-K, \\
& F\left(x_{n-1}, x_{n}\right)+\psi\left(x_{n-1}\right) q\left(x_{n-1}, x_{n}\right) k_{0} \subset-K .
\end{aligned}
$$

Adding the above $n$ inclusions up, we have

$$
\sum_{i=1}^{n} F\left(x_{i-1}, x_{i}\right)+\sum_{i=1}^{n} \psi\left(x_{i-1}\right) q\left(x_{i-1}, x_{i}\right) k_{0} \subset-K .
$$

By condition (iii),

$$
F\left(x_{0}, x_{n}\right) \subset \sum_{i=1}^{n} F\left(x_{i-1}, x_{i}\right)-K .
$$

Since $F\left(x_{i-1}, x_{i}\right) \subset-K$, we have $\psi\left(x_{i}\right) \geq \psi\left(x_{i-1}\right), i=1,2, \ldots, n$. Thus,

$$
\psi\left(x_{0}\right) \sum_{i=1}^{n} q\left(x_{i-1}, x_{i}\right) k_{0} \subset \sum_{i=1}^{n} \psi\left(x_{i-1}\right) q\left(x_{i-1}, x_{i}\right) k_{0}-K .
$$

Combining (3.3), (3.4) and (3.2), we have

$$
\begin{aligned}
& F\left(x_{0}, x_{n}\right)+\psi\left(x_{0}\right) \sum_{i=1}^{n} q\left(x_{i-1}, x_{i}\right) k_{0} \\
& \quad \subset \sum_{i=1}^{n} F\left(x_{i-1}, x_{i}\right)+\sum_{i=1}^{n} \psi\left(x_{i-1}\right) q\left(x_{i-1}, x_{i}\right) k_{0}-K \\
& \quad \subset-K-K \\
& \quad=-K .
\end{aligned}
$$

Thus,

$$
F\left(x_{0}, x_{n}\right)+s_{0}+\psi\left(x_{0}\right) \sum_{i=1}^{n} q\left(x_{i-1}, x_{i}\right) k_{0} \subset s_{0}-K .
$$

Take any $y_{n} \in F\left(x_{0}, x_{n}\right)$. By condition (ii), $y_{n} \notin-s_{0}+\alpha k_{0}-K$. Thus,

$$
\xi_{k_{0}}\left(y_{n}+s_{0}\right) \geq \alpha
$$

By (3.5) and Proposition 2.3, we have

$$
\xi_{k_{0}}\left(y_{n}+s_{0}\right)+\psi\left(x_{0}\right) \sum_{i=1}^{n} q\left(x_{i-1}, x_{i}\right) \leq \xi_{k_{0}}\left(s_{0}\right)<+\infty .
$$


By (3.7) and (3.6), we have

$$
\sum_{i=1}^{n} q\left(x_{i-1}, x_{i}\right) \leq \frac{1}{\psi\left(x_{0}\right)}\left(\xi_{k_{0}}\left(s_{0}\right)-\xi_{k_{0}}\left(y_{n}+s_{0}\right)\right) \leq \frac{1}{\psi\left(x_{0}\right)}\left(\xi_{k_{0}}\left(s_{0}\right)-\alpha\right)<+\infty, \forall n
$$

Hence

$$
\sum_{i=1}^{\infty} q\left(x_{i-1}, x_{i}\right) \leq \frac{1}{\psi\left(x_{0}\right)}\left(\xi_{k_{0}}\left(s_{0}\right)-\alpha\right)<+\infty .
$$

From this, for any $m>n$,

$$
q\left(x_{n}, x_{m}\right) \leq \sum_{i=n}^{m-1} q\left(x_{i}, x_{i+1}\right) \rightarrow 0 \quad(m>n \rightarrow \infty)
$$

Obviously, $\left\{x_{n}\right\}$ is a left-Cauchy sequence in $(X, q)$. Since $(X, q)$ is left-complete, there exists $\bar{x} \in X$ such that $q\left(x_{n}, \bar{x}\right) \rightarrow 0(n \rightarrow \infty)$. Remarking that $x_{n} \in S\left(x_{n-1}\right)$, we have $F\left(x_{n-1}, x_{n}\right) \subset-K, \forall n$. By condition (iv), we have

$$
F\left(x_{n-1}, \bar{x}\right) \subset-K, \forall n .
$$

For any fixed $n$, when $m>n$, we have $x_{m} \in S\left(x_{n}\right)$, i.e.

$$
F\left(x_{n}, x_{m}\right)+\psi\left(x_{n}\right) q\left(x_{n}, x_{m}\right) k_{0} \subset-K .
$$

By condition (iii), (3.8) and (3.9), we have

$$
\begin{aligned}
F & \left(x_{n}, \bar{x}\right)+\psi\left(x_{n}\right) q\left(x_{n}, x_{m}\right) k_{0} \\
& \subset F\left(x_{n}, x_{m}\right)+F\left(x_{m}, \bar{x}\right)-K+\psi\left(x_{n}\right) q\left(x_{n}, x_{m}\right) k_{0} \\
& \subset-K-K-K \\
& =-K .
\end{aligned}
$$

From (3.10), we have

$$
\begin{aligned}
& F\left(x_{n}, \bar{x}\right)+\psi\left(x_{n}\right) q\left(x_{n}, \bar{x}\right) k_{0} \\
& \quad=F\left(x_{n}, \bar{x}\right)+\psi\left(x_{n}\right) q\left(x_{n}, x_{m}\right) k_{0}+\psi\left(x_{n}\right)\left(q\left(x_{n}, \bar{x}\right)-q\left(x_{n}, x_{m}\right)\right) k_{0} \\
& \quad \subset-K++\psi\left(x_{n}\right)\left(q\left(x_{n}, \bar{x}\right)-q\left(x_{n}, x_{m}\right)\right) k_{0} \\
& \quad \subset-K+\psi\left(x_{n}\right) q\left(x_{m}, \bar{x}\right) k_{0} .
\end{aligned}
$$

Since $q\left(x_{m}, \bar{x}\right) \rightarrow 0(m \rightarrow \infty)$ and $K$ is $k_{0}$-closed, by Proposition 2.1 we have

$$
F\left(x_{n}, \bar{x}\right)+\psi\left(x_{n}\right) q\left(x_{n}, \bar{x}\right) k_{0} \subset \cap_{m>n}\left(-K+\psi\left(x_{n}\right) q\left(x_{m}, \bar{x}\right) k_{0}\right)=-K .
$$

That is, $\bar{x} \in S\left(x_{n}\right), \forall n$. Thus, condition (C) is satisfied.

In Theorem 3.1, we needn't assume that the ordering cone $K$ is closed, we only assume that $K$ is $k_{0}$-closed. The usual assumption on lower semi-continuity of objective map is replaced by a weaker one: $F$ is left $K$-slm in $(X, q)$. And the assumption on lower boundedness of $F\left(x_{0}, X\right)$ is taking the weakest form as shown in condition (ii) (please refer to [18,21,22]). As pointed out in [47], condition (iv) in Theorem 3.1 can be weakened to the so-called limiting monotonicity property of the set-valued map $S(\cdot)$ (see [5]), known also as dynamical closedness in [19].

Definition 6 (refer to [19]): Let $(X, q)$ be a quasi-metric space and let $S: X \rightarrow 2^{X} \backslash\{\emptyset\}$ be a setvalued map. The set-valued map $S(\cdot)$ is said to be dynamically left-closed at $x \in X$ in $(X, q)$ iff for 
any sequence $\left\{x_{n}\right\} \subset S(x)$ such that $x_{n+1} \in S\left(x_{n}\right) \subset S(x), \forall n$, and $q\left(x_{n}, \bar{x}\right) \rightarrow 0(n \rightarrow \infty)$, one has $\bar{x} \in S(x)$. In this case, we also say that $S(x)$ is dynamically left-closed in $(X, q)$. Moreover, the set-valued map $S(\cdot)$ is said to be dynamically left-complete at $x \in X$ in $(X, q)$ iff for any sequence $\left\{x_{n}\right\} \subset S(x)$ such that $x_{n+1} \in S\left(x_{n}\right) \subset S(x), \forall n$, and $q\left(x_{n}, x_{m}\right) \rightarrow 0(m>n \rightarrow \infty)$, there exists $\bar{x} \in S(x)$ such that $q\left(x_{n}, \bar{x}\right) \rightarrow 0(n \rightarrow \infty)$. In this case we also say that $S(x)$ is dynamically left-complete in $(X, q)$.

Theorem 3.2: $\quad$ Let $(X, q)$ be a left-complete quasi-metric space, $Y$ be a real linear space, $K \subset Y$ be a convex cone and $k_{0} \in K \backslash-\operatorname{vcl}(K)$ such that $K$ is $k_{0}$-closed. Let $F: X \times X \rightarrow 2^{Y} \backslash\{\emptyset\}$ be a set-valued bimap satisfying the following conditions:

(i) $\exists x_{0} \in X$ such that $F\left(x_{0}, x_{0}\right) \subset-K$;

(ii) $\exists \alpha \in \mathbf{R}, \exists s_{0} \in(-\infty,+\infty) k_{0}-K$ such that

$$
F\left(x_{0}, X\right) \cap\left(-s_{0}+\alpha k_{0}-K\right)=\emptyset ;
$$

(iii) $F(x, z) \subset F(x, y)+F(y, z)-K, \forall x, y, z \in X$;

(iv) $S(\cdot): X \rightarrow 2^{X} \backslash\{\emptyset\}$ is dynamically left-closed at every $x \in S\left(x_{0}\right)$, where $S(x):=\left\{x^{\prime} \in X\right.$ : $\left.F\left(x, x^{\prime}\right)+\psi(x) q\left(x, x^{\prime}\right) k_{0} \subset-K\right\}$ and $\psi \in \Psi_{F}$.

Then, there exists $\bar{x} \in X$ such that

(a) $F\left(x_{0}, \bar{x}\right)+\psi\left(x_{0}\right) q\left(x_{0}, \bar{x}\right) k_{0} \subset-K$;

(b) $\forall x \in X \backslash\{\bar{x}\}, F(\bar{x}, x)+\psi(\bar{x}) q(\bar{x}, x) k_{0} \not \subset-K$.

Proof: We only need to prove that condition (C) in Theorem 3.A is satisfied. Let a sequence $\left\{x_{n}\right\} \subset$ $S\left(x_{0}\right)$ such that $x_{n} \in S\left(x_{n-1}\right), \forall n$. As done in the proof of Theorem 3.1, we have

$$
\sum_{i=1}^{\infty} q\left(x_{i-1}, x_{i}\right) \leq \frac{1}{\psi\left(x_{0}\right)}\left(\xi_{k_{0}}\left(s_{0}\right)-\alpha\right)<+\infty .
$$

From this, we know that $\left\{x_{n}\right\}$ is a left-Cauchy sequence in $(X, q)$. Since $(X, q)$ is left-complete, there exists $\bar{x} \in X$ such that $q\left(x_{n}, \bar{x}\right) \rightarrow 0(n \rightarrow \infty)$. For any given $n, q\left(x_{n+p}, \bar{x}\right) \rightarrow 0(p \rightarrow \infty)$ and $x_{n+p} \in S\left(x_{n+p-1}\right) \subset S\left(x_{n}\right)$, we have $\bar{x} \in S\left(x_{n}\right)$ since $S\left(x_{n}\right)$ is dynamically left-closed.

Theorem 3.3: Let $(X, q)$ be a left-Hausdorff (see [52,55]) quasi-metric space and let $Y, K, k_{0}$ be the same as in Theorem 3.2. Let $F: X \times X \rightarrow 2^{Y} \backslash\{\emptyset\}$ be a set-valued bimap satisfying conditions (i), (ii), (iii) in Theorem 3.2 and the following condition:

(iv)" $S(\cdot): X \rightarrow 2^{X} \backslash\{\emptyset\}$ is dynamically left-complete at every $x \in S\left(x_{0}\right)$, where $S(\cdot)$ is the same as in Theorem 3.2 .

Then, there exists $\bar{x} \in X$ such that

(a) $F\left(x_{0}, \bar{x}\right)+\psi\left(x_{0}\right) q\left(x_{0}, \bar{x}\right) k_{0} \subset-K$;

(b) $\forall x \in X \backslash\{\bar{x}\}, F(\bar{x}, x)+\psi(\bar{x}) q(\bar{x}, x) k_{0} \not \subset-K$.

Proof: We only need to prove that condition (C) in Theorem 3.A is satisfied. Let a sequence $\left\{x_{n}\right\} \subset$ $S\left(x_{0}\right)$ such that $x_{n} \in S\left(x_{n-1}\right), \forall n$. As done in the proof of Theorem 3.2, we know that $\left\{x_{n}\right\}$ is a left-Cauchy sequence in $(X, q)$. For any fixed $n,\left\{x_{n+p}\right\}_{p \in \mathbf{N}} \subset S\left(x_{n}\right)$ and $x_{n+p} \in S\left(x_{n+p-1}\right)$ and $\left\{x_{n+p}\right\}_{p \in \mathbf{N}}$ is a left-Cauchy sequence in $(X, q)$. By condition (iv) $)^{\prime \prime}, S(\cdot)$ is dynamically left-complete at $x_{n} \in S\left(x_{0}\right)$, so there exists $\bar{x} \in S\left(x_{n}\right)$ such that $q\left(x_{n+p}, \bar{x}\right) \rightarrow 0(p \rightarrow \infty)$. Since $(X, q)$ is leftHausdorff, $\bar{x}$ is uniquely determined and $\bar{x} \in S\left(x_{n}\right), \forall n$. That is, condition (C) in Theorem 3.A is satisfied. 
Combining Theorem 3.1 and Proposition 2.6, we immediately obtain the following result, which extends [32, Theorem 3.1], [37, Theorem 3.1] and [36, Theorem 4.1].

Theorem 3.4: Let $(X, q)$ be a left-complete quasi-metric space, $Y$ be a t.v.s., $K \subset Y$ be a closed convex cone and $k_{0} \in K \backslash-K$. Let $F: X \times X \rightarrow 2^{Y} \backslash\{\emptyset\}$ be a set-valued bimap satisfying the following conditions:

(i) $\exists x_{0} \in X$ such that $F\left(x_{0}, x_{0}\right) \subset-K$;

(ii) $\exists \alpha \in \mathbf{R}, \exists s_{0} \in(-\infty,+\infty) k_{0}-K$ such that

$$
F\left(x_{0}, X\right) \cap\left(-s_{0}+\alpha k_{0}-K\right)=\emptyset ;
$$

(iii) $F(x, z) \subset F(x, y)+F(y, z)-K, \forall x, y, z \in X$;

(iv) $F$ is left $K$-slm in $(X, q)$; or for each $x \in X$, the map: $y \mapsto F(x, y)$ is quasi left-K-lower semi-continuous, i.e. for any $b \in Y,\{y \in X: F(x, y) \subset b-K\}$ is left-closed.

Then, for any $\psi \in \Psi_{F}$, there exists $\bar{x} \in X$ such that

(a) $F\left(x_{0}, \bar{x}\right)+\psi\left(x_{0}\right) q\left(x_{0}, \bar{x}\right) k_{0} \subset-K$;

(b) $\forall x \in X \backslash\{\bar{x}\}, F(\bar{x}, x)+\psi(\bar{x}) q(\bar{x}, x) k_{0} \not \subset-K$.

Condition (ii) in Theorems 3.1-3.4 is strictly weaker than condition (ii) in [32, Theorem 3.1] and condition (ii) in [30, Theorem 1], i.e. there exists $k^{*} \in K^{+}$with $k^{*}\left(k_{0}\right)>0$ such that $k^{*} \circ F\left(x_{0}, \cdot\right)$ is lower bounded. Here, $K^{+}$denotes the dual cone $\left\{y^{*} \in Y^{*}: y^{*}(k) \geq 0, \forall k \in K\right\}$ and $Y^{*}$ is the topological dual of $Y$. For convenience, instead of $F\left(x_{0}, \cdot\right)$ we consider a set-valued map $f: X \rightarrow 2^{Y} \backslash\{\emptyset\}$, Assume that there exists $k^{*} \in K^{+}$with $k^{*}\left(k_{0}\right)>0$ such that $k^{*}(f(X))$ is lower bounded, i.e. there exists $\gamma \in \mathbf{R}$ such that

$$
\gamma \leq k^{*}(y), \forall x \in X, \forall y \in f(x)
$$

In fact, (3.11) implies that for any given $t_{0} \in Y$, there exists $\epsilon>0$ such that $f(X) \cap\left(t_{0}-\epsilon k_{0}-K\right)=\emptyset$. If not, for every $n, f(X) \cap\left(t_{0}-n k_{0}-K\right) \neq \emptyset$. Thus, for each $n, \exists x_{n} \in X, \exists y_{n} \in f\left(x_{n}\right), \exists k_{n} \in K$ such that

$$
y_{n}=t_{0}-n k_{0}-k_{n}
$$

From this, we have

$$
k^{*}\left(y_{n}\right) \leq k^{*}\left(t_{0}\right)-n k^{*}\left(k_{0}\right), \quad \forall n .
$$

Combining this with (3.11), we have

$$
-\infty<\gamma-k^{*}\left(t_{0}\right) \leq k^{*}\left(y_{n}\right)-k^{*}\left(t_{0}\right) \leq-n k^{*}\left(k_{0}\right), \quad \forall n,
$$

where $k^{*}\left(k_{0}\right)>0$. This is impossible. The following example shows that the converse of the above implication is not true.

Example 1: Let $X$ be $\mathbf{R}$ endowed with the usual metric, $Y$ be $\mathbf{R}^{2}$ endowed with the usual topology, the closed convex cone $K \subset Y$ be $\left\{\left(y_{1}, y_{2}\right) \in \mathbf{R}^{2}: y_{1} \geq 0, y_{2} \geq 0\right\}$ and $k_{0} \in K \backslash-K$ be the point $(1,1) \in Y=\mathbf{R}^{2}$. A set-valued map $f: X \rightarrow 2^{Y} \backslash\{\emptyset\}$ is defined as follows:

$$
f(x)=\{(x, 1),(0, x)\} \subset Y, \quad x \in X .
$$

It is easy to verify that

$$
f(X) \cap\left(-k_{0}-K\right)=\emptyset .
$$

But, for any $y^{*} \in Y^{*} \backslash\{0\}, y^{*}(f(X))$ is not lower bounded.

In Theorems 3.1-3.4, if the 'left' is replaced by the 'right', we can obtain the corresponding four theorems. Here, we won't write the details. 
Particularly, if $F(x, y)$ is a singleton for every $(x, y) \in X \times X$, i.e. $F: X \times X \rightarrow Y$ is a vectorvalued bimap, then from Theorems 3.1-3.4, we can obtain the corresponding equilibrium versions of vector-valued EVP in quasi-metric spaces. Since the case of vector-valued maps is easier to handle than the case of set-valued maps, we even can obtain better results.

Corollary 3.5: Let $(X, q)$ be a left-complete quasi-metric space, $Y$ be a real linear space, $K \subset Y$ be a convex cone and $k_{0} \in K \backslash-\operatorname{vcl}(K)$ such that $K$ is $k_{0}$-closed. Let $F: X \times X \rightarrow Y$ be a vector-valued bimap satisfying the following conditions:

(i) $F(x, x) \in-K, \forall x \in X$;

(ii) $\exists x_{0} \in X, \exists \alpha \in \mathbf{R}, \exists s_{0} \in(-\infty,+\infty) k_{0}-K$ such that

$$
F\left(x_{0}, X\right) \cap\left(-s_{0}+\alpha k_{0}-K\right)=\emptyset ;
$$

(iii) $F(x, z) \in F(x, y)+F(y, z)-K, \forall x, y, z \in X$;

(iv) $F$ is left $K$-slm in $(X, q)$.

Then, for any $\psi \in \Psi_{F}$ and any $y_{0} \in X$ such that $F\left(x_{0}, y_{0}\right) \in(-\infty,+\infty) k_{0}-K$, there exists $\bar{y} \in X$ such that

(a) $F\left(y_{0}, \bar{y}\right)+\psi\left(x_{0}\right) q\left(y_{0}, \bar{y}\right) k_{0} \in-K$;

(b) $\forall y \in X \backslash\{\bar{y}\}, F(\bar{y}, y)+\psi(\bar{x}) q(\bar{y}, y) k_{0} \notin-K$.

Proof: For any $x \in X$, by (iii) we have

$$
F\left(x_{0}, x\right) \leq_{K} F\left(x_{0}, y_{0}\right)+F\left(y_{0}, x\right)
$$

and

$$
F\left(x_{0}, x\right)+s_{0} \leq_{K} F\left(x_{0}, y_{0}\right)+F\left(y_{0}, x\right)+s_{0} .
$$

By Proposition 2.3 we have

$$
\xi_{k_{0}}\left(F\left(x_{0}, x\right)+s_{0}\right) \leq \xi_{k_{0}}\left(F\left(x_{0}, y_{0}\right)\right)+\xi_{k_{0}}\left(F\left(y_{0}, x\right)+s_{0}\right)
$$

By (ii), $\xi_{k_{0}}\left(F\left(x_{0}, x\right)+s_{0}\right)>\alpha$. Combining this with (3.12), we have

$$
\xi_{k_{0}}\left(F\left(y_{0}, x\right)+s_{0}\right) \geq \xi_{k_{0}}\left(F\left(x_{0}, x\right)+s_{0}\right)-\xi_{k_{0}}\left(F\left(x_{0}, y_{0}\right)\right)>\alpha-\xi_{k_{0}}\left(F\left(x_{0}, y_{0}\right)\right),
$$

where $\xi_{k_{0}}\left(F\left(x_{0}, y_{0}\right)\right)$ is a usual real number since $F\left(x_{0}, y_{0}\right) \in(-\infty,+\infty) k_{0}-K$. Put $\alpha^{\prime}=\alpha-$ $\xi_{k_{0}}\left(F\left(x_{0}, y_{0}\right)\right)$. Then,

$$
F\left(y_{0}, X\right) \cap\left(-s_{0}+\alpha^{\prime} k_{0}-K\right)=\emptyset .
$$

Now, substituting $x_{0}$ by $y_{0}$ in Theorem 3.1, we obtain $\bar{y} \in X$ such that (a) and (b) hold.

Similarly, from Theorems 3.2 and 3.3 we have the following results.

Corollary 3.6: Let $(X, q), Y, K, k_{0}$ be the same as in Corollary 3.5. Let $F: X \times X \rightarrow Y$ be a vector-valued bimap satisfying conditions (i), (ii), (iii) in Corollary 3.5 and the following condition

(iv) $)^{\prime} \quad S(\cdot): X \rightarrow 2^{X} \backslash\{\emptyset\}$ is dynamically left-closed at every $x \in X$, where $S(x):=\left\{x^{\prime} \in X:\right.$ $\left.F\left(x, x^{\prime}\right)+\psi(x) q\left(x, x^{\prime}\right) k_{0} \in-K\right\}$ and $\psi \in \Psi_{F}$.

Then, for any $y_{0} \in X$ such that $F\left(x_{0}, y_{0}\right) \in(-\infty,+\infty) k_{0}-K$, there exists $\bar{y} \in X$ such that (a) and (b) hold in Corollary 3.5.

Corollary 3.7: Let $(X, q)$ be a left-Hausdorff quasi-metric space and let $Y, K, k_{0}$ be the same as in Corollary 3.5. Let $F: X \times X \rightarrow Y$ be a vector-valued map satisfying conditions (i), (ii), (iii) in Corollary 3.5 and the following condition 
(iv)" $S(\cdot): X \rightarrow 2^{X} \backslash\{\emptyset\}$ is dynamically left-complete at every $x \in X$, where $S(x):=\left\{x^{\prime} \in X:\right.$ $\left.F\left(x, x^{\prime}\right)+\psi(x) q\left(x, x^{\prime}\right) k_{0} \in-K\right\}$ and $\psi \in \Psi_{F}$.

Then, for any $y_{0} \in X$ such that $F\left(x_{0}, y_{0}\right) \in(-\infty,+\infty) k_{0}-K$, there exists $\bar{y} \in X$ such that (a) and (b) hold in Corollary 3.5.

If $Y$ is a t.v.s., $K \subset Y$ is a closed convex cone and $k_{0} \in \operatorname{int}(K)$, then $k_{0}-K$ is an absorbing set and the assumption that $F\left(x_{0}, y_{0}\right) \in(-\infty,+\infty) k_{0}-K$ in Corollaries 3.5-3.7. is automatically satisfied. Particularly, from Corollary 3.5, we can obtain the following corollary, which extends and improves [30, Theorem 1].

Corollary 3.8: Let $(X, q)$ be a left-complete quasi-metric space, $Y$ be a t.v.s., $K \subset Y$ be a closed convex cone and $k_{0} \in \operatorname{int}(K)$. Let $F: X \times X \rightarrow Y$ be a vector-valued bimap satisfying the following conditions:

(i) $F(x, x) \in-K, \forall x \in X$;

(ii) $\exists x_{0} \in X, \exists \alpha \in \mathbf{R}, \exists t_{0} \in Y$ such that

$$
F\left(x_{0}, X\right) \cap\left(t_{0}+\alpha k_{0}-K\right)=\emptyset
$$

(iii) $F(x, z) \in F(x, y)+F(y, z)-K, \forall x, y, z \in X$;

(iv) $F$ is left $K$-slm in $(X, q)$; or, for each $x \in X$, the map: $y \mapsto F(x, y)$ is left-quasi $K$-lower semi-continuous (see Proposition 2.6).

Then, for any $\psi \in \Psi_{F}$ and any $y_{0} \in X$, there exists $\bar{y} \in X$ such that

(a) $F\left(y_{0}, \bar{y}\right)+\psi\left(y_{0}\right) q\left(y_{0}, \bar{y}\right) k_{0} \in-K$;

(b) $\forall y \in X \backslash\{\bar{y}\}, F(\bar{y}, y)+\psi(\bar{y}) q(\bar{y}, y) k_{0} \notin-K$.

Remark 2: It seems that Corollary 3.8 is a particular case of Theorem 3.4. We remark that the requirements on lower boundedness for objective bimap in Corollary 3.8 and in Theorem 3.4 are similar. But, the result of Corollary 3.8 is more flexible. It points out that for any $y_{0} \in X$, there exists $\bar{y} \in X$ such that (a) and (b) hold. Here, the point $y_{0}$ is arbitrary, it is not confined to the point $x_{0}$.

More particularly, if there exists a vector-valued map $f$ such that the bimap $F$ can be written as the following form: $F(x, y)=f(y)-f(x), \forall x, y \in X$, then from Corollary 3.5 and its right version, we can obtain the following corollary, which extends [41, Theorem 2.4], [45, Corollary 3.3], [48, Theorem 4.8] and [47, Theorem 3.2] to the case of vector-valued maps.

Corollary 3.9: $\quad$ Let $(X, q)$ be a left-complete quasi-metric space, $Y$ be a t.v.s., $K \subset Y$ be a closed convex cone and $k_{0} \in K \backslash-K$. Let $f: X \rightarrow Y$ be a vector-valued map, $\epsilon>0$ and $x_{0} \in X$ such that

$$
\left(f\left(x_{0}\right)-\epsilon k_{0}-K\right) \cap f(X)=\emptyset .
$$

Case 1. If $(X, q)$ is left-complete and $f$ is left-K-slm, or $f$ is quasi left-K-lower semi-continuous, then for any $\lambda>0$, there exists $\bar{x} \in X$ such that
(a) $f(\bar{x})+\frac{\epsilon}{\lambda} q\left(x_{0}, \bar{x}\right) k_{0} \leq_{K} f\left(x_{0}\right)$;
(b) $q\left(x_{0}, \bar{x}\right) \leq \lambda$;
(c) $\forall x \in X \backslash\{\bar{x}\}, f(x)+\frac{\epsilon}{\lambda} q(\bar{x}, x) k_{0} \quad \bigsqcup_{K} f(\bar{x})$.

Case 2. If $(X, q)$ is right-complete and $f$ is right-K-slm, or $f$ is quasi right-K-lower semi-continuous, then for any $\lambda>0$, there exists $\bar{x} \in X$ such that
$\left(\mathrm{a}^{\prime}\right) f(\bar{x})+\frac{\epsilon}{\lambda} q\left(\bar{x}, x_{0}\right) k_{0} \leq_{K} f\left(x_{0}\right)$
(b') $q\left(\bar{x}, x_{0}\right) \leq \lambda$;
(c') $\forall x \in X \backslash\{\bar{x}\}, f(x)+\frac{\epsilon}{\lambda} q(x, \bar{x}) k_{0} \bigsqcup_{K} f(\bar{x})$. 
Proof: We only consider Case 1 . Put $F(x, y)=f(y)-f(x), \forall x, y \in X$. Obviously, $F$ satisfies conditions (i) and (ii) in Corollary 3.5. The assumption that

$$
\left(f\left(x_{0}\right)-\epsilon k_{0}-K\right) \cap f(X)=\varnothing
$$

is equivalent to that

$$
F\left(x_{0}, X\right) \cap\left(-\epsilon k_{0}-K\right)=\emptyset .
$$

Thus, condition (ii) in Corollary 3.5 is satisfied, where $s_{0}=0$ and $\alpha=-\epsilon$. Also, $f$ being left- $K$-slm implies that $F$ is left- $K$-slm and condition (iv) in Corollary 3.5 is satisfied. For any $\lambda>0$, we take $\psi=\epsilon / \lambda$ a constant. Now, applying Corollary 3.5, we conclude that there exists $\bar{x} \in X$ such that

$$
F\left(x_{0}, \bar{x}\right)+\frac{\epsilon}{\lambda} q\left(x_{0}, \bar{x}\right) k_{0} \leq_{K} 0
$$

and

$$
\forall x \in X \backslash\{\bar{x}\}, F(\bar{x}, x)+\frac{\epsilon}{\lambda} q(\bar{x}, x) k_{0} \bigsqcup_{K} 0 .
$$

From this, we know that (a) and (b) hold.

Assume that $q\left(x_{0}, \bar{x}\right)>\lambda$. Then, by (a) we have

$$
f(\bar{x}) \in f\left(x_{0}\right)-\frac{\epsilon}{\lambda} q\left(x_{0}, \bar{x}\right) k_{0}-K \subset f\left(x_{0}\right)-\epsilon k_{0}-K,
$$

which contradicts the assumption that

$$
\left(f\left(x_{0}\right)-\epsilon k_{0}-K\right) \cap f(X)=\emptyset .
$$

Thus, (b) is proved.

In a similar way, we can prove the result in Case 2.

Similarly, from Corollary 3.6, we can deduce the following corollary, which extends [47, Theorem 3.3] to the case of vector-valued maps.

Corollary 3.10: Let $(X, q)$ be a left-complete quasi-metric space, $Y$ be a t.v.s., $K \subset Y$ be a closed convex cone and $k_{0} \in \operatorname{int}(K)$. Let $f: X \rightarrow Y$ be a vector-valued map satisfying the following conditions:

(i) $\exists t_{0} \in Y, \exists \alpha \in \mathbf{R}$ such that

$$
f(X) \cap\left(t_{0}+\alpha k_{0}-K\right)=\emptyset ;
$$

(ii) $S(\cdot): X \rightarrow 2^{X} \backslash\{\emptyset\}$ is dynamically left-closed at every $x \in X$, where $S(x):=\left\{x^{\prime} \in X\right.$ : $\left.f\left(x^{\prime}\right)+\psi(x) q\left(x, x^{\prime}\right) k_{0} \leq_{K} f(x)\right\}$ and $\psi \in \Psi_{F}$.

Then, for any $x_{0} \in X$, there exists $\bar{x} \in X$ such

(a) $f(\bar{x})+\psi\left(x_{0}\right) q\left(x_{0}, \bar{x}\right) k_{0} \leq_{K} f\left(x_{0}\right)$;

(b) $\forall x \in X \backslash\{\bar{x}\}, f(x)+\psi(\bar{x}) q(\bar{x}, x) k_{0} \bigsqcup_{K} f(\bar{x})$.

\section{Applications to equilibrium problems, vector optimization and fixed point theorems}

In this section, we shall apply equilibrium versions of EVP in quasi-metric spaces to equilibrium problems, optimization and fixed point theorems. 
Let $(X, q)$ be a quasi-metric space, $Y$ be a t.v.s. quasi-ordered by a closed convex cone $K$ with $\operatorname{int}(K) \neq \varnothing$, and let $F: X \times X \rightarrow 2^{Y} \backslash\{\varnothing\}$ be a set-valued bimap. We consider the set-valued equilibrium problem (denoted by SEP): finding $\bar{x} \in X$ such that

$$
F(\bar{x}, y) \not \subset-\operatorname{int}(K), \forall y \in X .
$$

A point $\bar{x}$ satisfying (4.1) is called a solution (or a equilibrium point) for SEP.

Particularly, if $F: X \times X \rightarrow Y$ is a vector-valued bimap, we consider the vector equilibrium problem (denoted by VEP): finding $\bar{x} \in X$ such that

$$
F(\bar{x}, y) \notin-\operatorname{int}(K), \quad \forall y \in X
$$

A point $\bar{x}$ satisfying (4.2) is called a solution (or a equilibrium point) for VEP.

More particularly, if $f: X \rightarrow Y$ is a vector-valued map and $F(x, y)=f(y)-f(x), \forall x, y \in X$, then $\bar{x}$ is a weakly efficient solution for the vector optimization problem $\operatorname{Min}_{x \in X} f(x)$ iff $\bar{x}$ is a solution for VEP on $F$. In this case, (4.2) becomes

$$
F(\bar{x}, y)=f(y)-f(\bar{x}) \notin-\operatorname{int}(K), \forall y \in X .
$$

Definition 7: A quasi-metric space $(X, q)$ is said to be left-sequentially compact, iff for every sequence $\left\{x_{n}\right\} \subset X$, there exists a subsequence $\left\{x_{n_{i}}\right\}$ and a point $\bar{x} \in X$ such that $q\left(x_{n_{i}}, \bar{x}\right) \rightarrow$ $0(i \rightarrow \infty)$.

Lemma 4.1: If a quasi-metric space $(X, q)$ is left-sequentially compact, then it is left-complete.

Proof: Let $\left\{x_{n}\right\} \subset X$ be a left-Cauchy sequence. For any given $\epsilon>0$, there exists $N \in \mathbf{N}$ such that

$$
q\left(x_{m}, x_{p}\right)<\epsilon, \forall p>m \geq N \text {. }
$$

Since $(X, q)$ is left-sequentially compact, there exists a subsequence $\left\{x_{n_{i}}\right\}$ and a point $\bar{x} \in X$ such that

$$
q\left(x_{n_{i}}, \bar{x}\right) \rightarrow 0(i \rightarrow \infty)
$$

For the above $\epsilon$ and above $m \geq N$, take $i_{0} \in \mathbf{N}$ such that

$$
q\left(x_{n_{i}}, \bar{x}\right)<\epsilon \text { and } n_{i}>m, \forall i \geq i_{0} .
$$

Thus, when $m \geq N$, we have

$$
q\left(x_{m}, \bar{x}\right) \leq q\left(x_{m}, x_{n_{i}}\right)+q\left(x_{n_{i}}, \bar{x}\right)<\epsilon+\epsilon=2 \epsilon .
$$

That is, $q\left(x_{m}, \bar{x}\right) \rightarrow 0(m \rightarrow \infty)$.

Theorem 4.2: $\quad$ Let $(X, q)$ be a left-sequentially compact quasi-metric space, $Y$ be a t.v.s., $K \subset Y$ be a closed convex solid cone and $k_{0} \in K \backslash-K$. Let $F: X \times X \rightarrow 2^{Y} \backslash\{\emptyset\}$ be a set-valued bimap satisfying the following conditions:

(i) $\exists x_{0} \in X$ such that $F\left(x_{0}, x_{0}\right) \subset-K$ and for every $x \in X, F(x, x) \cap(-\partial K) \neq \emptyset$;

(ii) $\exists \alpha \in \mathbf{R}, \exists s_{0} \in(-\infty,+\infty) k_{0}-K$ such that

$$
F\left(x_{0}, X\right) \cap\left(-s_{0}+\alpha k_{0}-K\right)=\emptyset
$$

(iii) $F(x, z) \subset F(x, y)+F(y, z)-K, \forall x, y, z \in X$; 
(iv) $F$ is left-slm, or for each $x \in X$, the map: $y \mapsto F(x, y)$ is quasi left-K-lower semi-continuous, i.e. for any $b \in Y,\{y \in X: F(x, y) \subset b-K\}$ is left-closed;

(v) for each $y \in X$, the map $x \mapsto F(x, y)$ is left-K-upper semi-continuous.

Moreover, for any $x, y \in X, F(x, y) \subset Y$ is a compact set.

Then, there exists $\bar{x} \in X$ such that

$$
F(\bar{x}, y) \not \subset-\operatorname{int}(K), \forall y \in X
$$

Proof: Obviously, conditions (i)-(iv) in Theorem 3.4 are satisfied. Taking $\psi \in \Psi_{F}$ to be a constant $\lambda=1 / n$, by Theorem 3.4 we can find $\bar{x}_{n} \in X$ such that

$$
F\left(\bar{x}_{n}, x\right)+\frac{1}{n} q\left(\bar{x}_{n}, x\right) k_{0} \not \subset-K, \forall x \in X \backslash\left\{\bar{x}_{n}\right\} .
$$

Since $(X, q)$ is left-sequentially compact, there exists a subsequence $\left\{\bar{x}_{n_{i}}\right\}$ of $\left\{\bar{x}_{n}\right\}$ and a point $\bar{x} \in X$ such that

$$
q\left(\bar{x}_{n_{i}}, \bar{x}\right) \rightarrow 0(i \rightarrow \infty)
$$

Briefly, we denote

$$
q\left(\bar{x}_{n}, \bar{x}\right) \rightarrow 0(n \rightarrow \infty)
$$

We assert that

$$
F(\bar{x}, y) \not \subset-\operatorname{int}(K), \forall y \in X .
$$

If not, there exists a point $\bar{y} \in X$ such that

$$
F(\bar{x}, \bar{y}) \subset-\operatorname{int}(K)
$$

Since $F(\bar{x}, \bar{y}) \subset Y$ is a compact set, there exists a circled neighbourhood $V$ of 0 in $Y$ (see [55]) such that

$$
F(\bar{x}, \bar{y})+V+V \subset-\operatorname{int}(K) .
$$

By (4.5), we have

$$
0 \leq q\left(\bar{x}_{n}, \bar{y}\right) \leq q\left(\bar{x}_{n}, \bar{x}\right)+q(\bar{x}, \bar{y}) \rightarrow q(\bar{x}, \bar{y})(n \rightarrow \infty) .
$$

Thus, there exists $N_{1} \in \mathbf{N}$ such that

$$
\frac{1}{n} q\left(\bar{x}_{n}, \bar{y}\right) k_{0} \in V, \forall n \geq N_{1} .
$$

By condition (v), $x \mapsto F(x, \bar{y})$ is left-K-upper semi-continuous at $\bar{x} \in X$ (see Definition 2). Hence, there exists a left-neighbourhood $U_{l}(\bar{x})$ of $\bar{x}$ such that

$$
F(x, \bar{y}) \subset F(\bar{x}, \bar{y})+V-K, \forall x \in U_{l}(\bar{x}) .
$$

Since $q\left(\bar{x}_{n}, \bar{x}\right) \rightarrow 0(n \rightarrow \infty)$, there exists $N_{2} \in \mathbf{N}$ such that

$$
\bar{x}_{n} \in U_{l}(\bar{x}), \quad \forall n \geq N_{2} .
$$

Thus,

$$
F\left(\bar{x}_{n}, \bar{y}\right) \subset F(\bar{x}, \bar{y})+V-K, \quad \forall n \geq N_{2} .
$$


Put $N=\max \left(N_{1}, N_{2}\right)$. Then, for $n \geq N$, by (4.8), (4.7) and (4.6), we have

$$
\begin{aligned}
F\left(\bar{x}_{n}, \bar{y}\right)+\frac{1}{n} q\left(\bar{x}_{n}, \bar{y}\right) k_{0} & \subset F(\bar{x}, \bar{y})+V-K+V \\
& \subset-\operatorname{int}(K)-K \\
& =-\operatorname{int}(K) .
\end{aligned}
$$

Assume that there exists $n$ such that $\bar{y}=\bar{x}_{n}$. Then, from (4.9) we have

$$
F(\bar{y}, \bar{y}) \subset-\operatorname{int}(K)
$$

which contradicts the assumption that $F(\bar{y}, \bar{y}) \cap(-\partial K) \neq \emptyset$. Therefore, for every $n, \bar{y} \neq \bar{x}_{n}$. Thus, (4.9) contradicts (4.4).

Obviously, Theorem 4.2 extends and improves [32, Theorem 4.1] and [37, Theorem 4.1]. Particularly, if $F$ is a vector-valued bimap, then from Theorem 4.2 we can obtain the following result, which extends and improves [27, Theorem 5.1], [29, Proposition 3.2], [30, Theorem 3] and [31, Theorem 2.2].

Theorem 4.3: $\quad$ Let $(X, q)$ be a left-sequentially compact quasi-metric space, $Y$ be a t.v.s., $K \subset Y$ be a closed convex solid cone and $k_{0} \in K \backslash-K$. Let $F: X \times X \rightarrow Y$ be a vector-valued bimap satisfying the following conditions:

(i) $F(x, x) \in-\partial K, \forall x \in X$;

(ii) $\exists x_{0} \in X, \exists \alpha \in \mathbf{R}, \exists s_{0} \in(-\infty,+\infty) k_{0}-K$ such that

$$
F\left(x_{0}, X\right) \cap\left(-s_{0}+\alpha k_{0}-K\right)=\emptyset
$$

(iii) $F(x, z) \leq_{K} F(x, y)+F(y, z), \forall x, y, z \in X$;

(iv) $F$ is left-slm, or for each $x \in X$, the map: $y \mapsto F(x, y)$ is quasi left-K-lower semi-continuous, i.e. for any $b \in Y,\{y \in X: F(x, y) \in b-K\}$ is left-closed;

(v) for each $y \in X$, the map $x \mapsto F(x, y)$ is left-K-upper semi-continuous.

Then, there exists $\bar{x} \in X$ such that

$$
F(\bar{x}, y) \notin-\operatorname{int}(K), \quad \forall y \in X
$$

Remark 3: If $k_{0}$ in Theorems 4.2 and 4.3 is exactly an interior point of $K$, i.e. $k_{0} \in \operatorname{int}(K)$, then condition (ii) can be rewritten as the following easier form:

$\exists x_{0} \in X, \exists \alpha \in \mathbf{R}, \exists t_{0} \in Y$ such that

$$
F\left(x_{0}, X\right) \cap\left(t_{0}+\alpha k_{0}-K\right)=\emptyset .
$$

More particularly, if $F(x, y)=f(y)-f(x), \forall x, y \in X$, where $f: X \rightarrow Y$ is a vector-valued map, then from Theorem 4.3 we can deduce the following existence result concerning solutions of vector optimization problem, which extends and improves [32, Corollary 4.1].

Theorem 4.4: Let $(X, q)$ be a left-sequentially compact quasi-metric space, $Y$ be a t.v.s., $K \subset Y$ be a closed convex solid cone and $k_{0} \in K \backslash-K$. Let $f: X \rightarrow Y$ be a left-K-lower semi-continuous map satisfying the following condition:

$\exists \alpha \in \mathbf{R}, \exists t_{0} \in Y$ such that

$$
f(X) \cap\left(t_{0}+\alpha k_{0}-K\right)=\varnothing .
$$


Then, there exists a weakly efficient solution of the vector optimization problem $\operatorname{Min}_{x \in X} f(x)$, i.e. there exists $\bar{x} \in X$ such that

$$
f(y) \notin f(\bar{x})-\operatorname{int}(K), \forall y \in X .
$$

Proof: Put $F(x, y)=f(y)-f(x), \forall x, y \in X$. Clearly, $F$ satisfies conditions (i)-(iii) in Theorem 4.3. Now, $f: X \rightarrow Y$ being left- $K$-lower semi-continuous means that for each $x \in X$, the map $y \mapsto F(x, y)$ is left- $K$-lower semi-continuous. From Remark 1 we see that this also means that for each $y \in X$, the map $x \mapsto F(x, y)$ is left- $K$-upper semi-continuous. Thus, conditions (iv) and (v) in Theorem 4.3 are also satisfied. Applying Theorem 4.3, we obtain the result immediately.

Applying the set-valued and vector-valued EVPs in Section 3, we can also obtain a number of new fixed point theorems. For example, applying Theorem 3.4, we obtain the following Caristi's fixed point theorem, which extends [25, Theorem 3] and [37, Theorem 5.1(3)].

Theorem 4.5: Let $(X, q)$ be a left-complete quasi-metric space, $Y$ be a t.v.s., $K \subset Y$ be a closed convex cone and $k_{0} \in \operatorname{int}(K)$. Let $F: X \times X \rightarrow 2^{Y} \backslash\{\emptyset\}$ be a set-valued bimap satisfying the following conditions:

(i) $\exists x_{0} \in X$ such that $F\left(x_{0}, x_{0}\right) \subset-K$;

(ii) $\exists \alpha \in \mathbf{R}, \exists t_{0} \in Y$ such that

$$
F\left(x_{0}, X\right) \cap\left(t_{0}+\alpha k_{0}-K\right)=\emptyset
$$

(iii) $F(x, z) \subset F(x, y)+F(y, z)-K, \forall x, y, z \in X$;

(iv) $F$ is left $K$-slm in $(X, q)$; or for each $x \in X$, the map: $y \mapsto F(x, y)$ is left-quasi $K$-lower semi-continuous, i.e. for any $b \in Y,\{y \in X: F(x, y) \subset b-K\}$ is left-closed.

Assume that $T: X \rightarrow 2^{X} \backslash\{\emptyset\}$ satisfies the following property

(T) $F(x, y)+\psi(x) q(x, y) k_{0} \subset-K, \forall x \in X, \forall y \in T x$, where $\psi \in \Psi_{F}$.

Then, there exists $\bar{x} \in X$ such that

(a) $F\left(x_{0}, \bar{x}\right)+\psi\left(x_{0}\right) q\left(x_{0}, \bar{x}\right) k_{0} \subset-K$;

(f) $T \bar{x}=\{\bar{x}\}$.

Assume that $T: X \rightarrow 2^{X} \backslash\{\emptyset\}$ satisfies the following property

( $\left.\mathrm{T}^{\prime}\right) \forall x \in X, \exists y \in T x$ such that $F(x, y)+\psi(x) q(x, y) k_{0} \subset-K$, where $\psi \in \Psi_{F}$.

Then, there exists $\bar{x} \in X$ such that

(a) $F\left(x_{0}, \bar{x}\right)+\psi\left(x_{0}\right) q\left(x_{0}, \bar{x}\right) k_{0} \subset-K$;

(f) $\bar{x} \in T \bar{x}$.

Proof: By Theorem 3.4, we conclude that there exists $\bar{x} \in X$ such that

(a) $F\left(x_{0}, \bar{x}\right)+\psi\left(x_{0}\right) q\left(x_{0}, \bar{x}\right) k_{0} \subset-K$;

(b)

$$
\forall x \in X \backslash\{\bar{x}\}, F(\bar{x}, x)+\psi(\bar{x}) q(\bar{x}, x) k_{0} \not \subset-K .
$$

Since $T$ satisfies property (T), for every $y \in T \bar{x}$, we have

$$
F(\bar{x}, y)+\psi(\bar{x}) q(\bar{x}, y) k_{0} \subset-K .
$$

Comparing (4.10) and (4.11), we have $y=\bar{x}$. Hence $T \bar{x}=\{\bar{x}\}$ and (f) holds.

Next, assume that $T$ satisfies property $\left(\mathrm{T}^{\prime}\right)$. For $\bar{x} \in X$, there exists $\bar{y} \in T \bar{x}$ such that

$$
F(\bar{x}, \bar{y})+\psi(\bar{x}) q(\bar{x}, \bar{y}) k_{0} \subset-K .
$$

Comparing (4.10) and (4.12), we have $\bar{y}=\bar{x}$. Hence, $\bar{x} \in T \bar{x}$ and $\left(f^{\prime}\right)$ holds. 
As we have seen, in Theorems 3.4 and 4.5 we require the bimap $F: X \times X \rightarrow 2^{Y} \backslash\{\emptyset\}$ satisfy the following four conditions:

(i) $\exists x_{0} \in X$ such that $F\left(x_{0}, x_{0}\right) \subset-K$;

(ii) $\exists \alpha \in \mathbf{R}, \exists t_{0} \in Y$ such that $F\left(x_{0}, X\right) \cap\left(t_{0}+\alpha k_{0}-K\right)=\emptyset$;

(iii) $F(x, z) \subset F(x, y)+F(y, z)-K, \forall x, y, z \in X$;

(iv) $F$ is left $K$-slm in $(X, q)$.

Let us recall the corresponding four conditions on $F$ in [37, Theorems 3.1 and 5.1]:

(i') $0 \in F(x, x), F(x, x) \subset-K, \forall x \in X$;

(ii') $\exists z \in Y$ such that $F(x, y) \subset z+K, \forall x, y \in X$;

(iii') $F(x, z) \subset F(x, y)+F(y, z)-K, \forall x, y, z \in X$;

(iv') $y \mapsto F(x, y)$ is quasi $K$-lower semi-continuous for all $x \in X$.

Comparing the above two groups of conditions on bimap $F$, at least in form we see that conditions (i), (ii) and (iv) are weaker than conditions (i'), (ii') and (iv'), respectively. Next, we give an example on bimap $F$, which satisfies conditions (i), (ii), (iii) and (iv); but doesn't satisfy (i'), (ii') and (iv'). For convenience, in our example, space $(X, q)$ is taken as a metric space.

Example 2: Let $X$ be the real number space $\mathbf{R}$ endowed with the usual metric and $Y$ be the real number space $\mathbf{R}$ endowed with the usual order (the ordering cone is $K=[0,+\infty)$ ) and endowed with the usual topology. Let $g: X \rightarrow 2^{Y} \backslash\{\varnothing\}$ be defined as follows:

$$
g(x)=\left\{\begin{array}{ccc}
\{x+1\} & \text { if } & 0<x<+\infty \\
{[1 / 2,1]} & \text { if } & x=0 \\
\{x /(1+|x|)\} & \text { if } & -\infty<x<0
\end{array}\right.
$$

Let a bimap $F: X \times X \rightarrow 2^{Y} \backslash\{\emptyset\}$ be defined as follows:

$$
F(x, y):=g(y)-g(x), \quad \forall x, y \in X
$$

Take $x_{0}=1 \in X$. Then,

$$
F\left(x_{0}, x_{0}\right)=g\left(x_{0}\right)-g\left(x_{0}\right)=\left\{x_{0}+1\right\}-\left\{x_{0}+1\right\}=\{0\} \subset-K .
$$

That is, condition (i) is satisfied. But, condition ( $\left.i^{\prime}\right)$ is not satisfied, since

$$
F(0,0)=g(0)-g(0)=\left[\frac{1}{2}, 1\right]-\left[\frac{1}{2}, 1\right] \not \subset-K=(-\infty, 0] .
$$

Take $t_{0}=0, \alpha=-5, k_{0}=1$. Then,

$$
\begin{aligned}
& F\left(x_{0}, X\right) \cap\left(t_{0}+\alpha k_{0}-K\right) \\
& \quad=\cup_{y \in X}\left(g(y)-g\left(x_{0}\right)\right) \cap(-5-K) \\
& \quad=\cup_{y \in X}(g(y)-2) \cap(-\infty,-5] .
\end{aligned}
$$

Remarking the expression of $g$, we have $(g(y)-2) \cap(-\infty,-5]=\varnothing$ for all $y \in X$, Hence $F\left(x_{0}, X\right) \cap\left(t_{0}+\alpha k_{0}-K\right)=\emptyset$. Thus, $F$ satisfies condition (ii). But, $F$ doesn't satisfy condition (ii'). Assume the contrary. There exists a $z \in Y=\mathbf{R}$ such that $F(x, y) \subset z+K, \forall x, y \in X$. That is,

$$
g(y)-g(x) \subset z+[0,+\infty), \forall x, y \in X
$$


Take $y=-1$ and take $x \in(0,+\infty)$. Then,

$$
F(x, y)=g(y)-g(x)=-\frac{1}{2}-(x+1), \quad \forall x>0 .
$$

If we take $x>0$ large enough, then we have $g(y)-g(x)<z$, which contradicts (4.13). Also, it is easy to see that $F$ satisfies condition (iii).

Finally, we show that $F$ satisfies condition (iv), but doesn't satisfy condition (iv' ${ }^{\prime}$ ). Let a sequence $\left\{x_{n}\right\} \subset X$ satisfy

$$
F\left(x_{n}, x_{n+1}\right)=g\left(x_{n+1}\right)-g\left(x_{n}\right) \subset-K, \forall n \text {, and } x_{n} \rightarrow \bar{x} \text { in } X .
$$

We can easily verify that for all $n, F\left(x_{n}, \bar{x}\right)=g(\bar{x})-g\left(x_{n}\right) \subset-K$ according to the following three cases: $\bar{x}>0, \bar{x}<0$, and $\bar{x}=0$.

Case 1. $\bar{x}>0$. Since $x_{n} \rightarrow \bar{x}$, without loss of generality we may assume that all $x_{n} \in(0,+\infty)$. By $g\left(x_{n+1}\right)-g\left(x_{n}\right) \subset-K$, we conclude that $x_{n+1} \leq x_{n}, \forall n$. Thus, $\bar{x} \leq x_{n}, \forall n$, and $g(\bar{x})-g\left(x_{n}\right) \subset-K$, i.e. $F\left(x_{n}, \bar{x}\right) \subset-K$.

Case 2. $\bar{x}<0$. Without loss of generality we may assume that all $x_{n} \in(-\infty, 0)$. By $g\left(x_{n+1}\right)-$ $g\left(x_{n}\right) \subset-K$, we conclude that $x_{n+1} \leq x_{n}, \forall n$. Thus, $\bar{x} \leq x_{n}, \forall n$, and $g(\bar{x})-g\left(x_{n}\right) \subset-K$, i.e. $F\left(x_{n}, \bar{x}\right) \subset-K$.

Case 3. $\bar{x}=0$. By $x_{n} \rightarrow \bar{x}=0$ and $g\left(x_{n+1}\right)-g\left(x_{n}\right) \subset-K$, we conclude that all $x_{n} \in(0,+\infty)$ and $x_{n+1} \leq x_{n}, \forall n$. Thus,

$$
g(\bar{x})-g\left(x_{n}\right)=[1 / 2,1]-\left(x_{n}+1\right) \subset-K, \text { i:e: } F\left(x_{n}, \bar{x}\right) \subset-K .
$$

Hence, we have shown that $F$ is $K$-slm, that is, $F$ satisfies (iv). But, $y \mapsto F(x, y)$ is not quasi $K$-lower semi-continuous, i.e. $F$ doesn't satisfy (iv'). In fact, let $x=0$ and let $b=-1 / 2$. Then,

$$
\begin{aligned}
\{y \in X: f(0, y) \subset b-K\} & =\{y \in X: g(y)-g(0) \subset b-K\} \\
& =\{y \in X: g(y)-[1 / 2,1] \subset-1 / 2-[0,+\infty)\} \\
& =(-\infty, 0),
\end{aligned}
$$

which is not closed in $X$. Thus, the map $y \mapsto f(0, y)$ is not quasi $K$-lower semi-continuous (see [37, Lemma 2.2]).

Similarly, using Corollaries 3.8 and 3.10, we can deduce the following versions of Caristi's fixed point theorem.

Theorem 4.6: Let $(X, q), Y, K, k_{0}$ be the same as in Theorem 4.5. Let $F: X \times X \rightarrow Y$ be a vector-valued bimap satisfying the following conditions:

(i) $F(x, x) \in-K, \forall x \in X$;

(ii) $\exists x_{0} \in X, \exists \alpha \in \mathbf{R}, \exists t_{0} \in Y$ such that

$$
F\left(x_{0}, X\right) \cap\left(t_{0}+\alpha k_{0}-K\right)=\emptyset ;
$$

(iii) $F(x, z) \in F(x, y)+F(y, z)-K, \forall x, y, z \in X$;

(iv) $F$ is left $K$-slm in $(X, q)$; or, for each $x \in X$, the map $y \mapsto F(x, y)$ is left-quasi $K$-lower semi-continuous.

Assume that $T: X \rightarrow 2^{X} \backslash\{\emptyset\}$ satisfies the following property

(T) $F(x, y)+\psi(x) q(x, y) k_{0} \in-K, \forall x \in X, \forall y \in T x$, where $\psi \in \Psi_{F}$.

Then, for any $y_{0} \in X$, there exists $\bar{y} \in X$ such that

(a) $F\left(y_{0}, \bar{y}\right)+\psi\left(y_{0}\right) q\left(y_{0}, \bar{y}\right) k_{0} \in-K$; 
(f) $T \bar{y}=\{\bar{y}\}$.

Assume that $T: X \rightarrow 2^{X} \backslash\{\emptyset\}$ satisfies the following property

( $\left.\mathrm{T}^{\prime}\right) \forall x \in X, \exists y \in T x$ such that $F(x, y)+\psi(x) q(x, y) k_{0} \in-K$, where $\psi \in \Psi_{F}$.

Then, for any $y_{0} \in X$, there exists $\bar{y} \in X$ such that

(a) $F\left(y_{0}, \bar{y}\right)+\psi\left(y_{0}\right) q\left(y_{0}, \bar{y}\right) k_{0} \in-K$;

(f) $\bar{y} \in T \bar{y}$.

Theorem 4.7: Let $(X, q), Y, K, k_{0}$ be the same as in Theorem 4.5. Let $\lambda>0$ be a constant and let $f: X \rightarrow Y$ be a vector-valued map satisfying the following conditions:

(i) $\exists \alpha \in \mathbf{R}, \exists t_{0} \in Y$ such that

$$
f(X) \cap\left(t_{0}+\alpha k_{0}-K\right)=\emptyset ;
$$

(ii) $f$ is left $K$-slm in $(X, q)$.

Assume that $T: X \rightarrow 2^{X} \backslash\{\varnothing\}$ satisfies the following property

(T) $f(y)+\lambda q(x, y) k_{0} \leq_{K} f(x), \forall x \in X, \forall y \in T x$.

Then, for any $x_{0} \in X$, there exists $\bar{x} \in X$ such that

(a) $f(\bar{x})+\lambda q\left(x_{0}, \bar{x}\right) k_{0} \leq_{K} f\left(x_{0}\right)$;

(f) $T \bar{x}=\{\bar{x}\}$.

Assume that $T: X \rightarrow 2^{X} \backslash\{\emptyset\}$ satisfies the following property

(f') $\forall x \in X, \exists y \in T x$ such that $f(y)+\lambda q(x, y) k_{0} \leq_{K} f(x)$.

Then, for any $x_{0} \in X$, there exists $\bar{x} \in X$ such that

(a) $f(\bar{x})+\lambda q\left(x_{0}, \bar{x}\right) k_{0} \leq_{K} f\left(x_{0}\right)$;

(f') $\bar{x} \in T \bar{x}$.

The proofs of Theorems 4.6 and 4.7 are similar to that of Theorem 4.5. Obviously, Theorem 4.7 extends [41, Theorem 2.12], [47, Theorem 4.4] and [43, Corollary 2(2)]. We remark that for rightcomplete quasi-metric spaces, we can also obtain the corresponding versions of Caristi's fixed point theorem. Here, we won't write the details.

\section{Applications to human dynamics: the existence and the robustness of traps}

\subsection{Robust traps and the variational rationality approach of human dynamics}

This paper belongs to a new line of research on variational principles and algorithms in optimization (dual descent methods, proximal algorithms, trust region methods, local search algorithms, robust optimization, ....) more oriented towards applications in human dynamics, using the recent (VR) variational rationality approach of human behaviours (Soubeyran, [49,50]). A human dynamic starts in an initial position, including individual or collective doings, havings or beings. Moving away from this position, it follows an acceptable transition, defined as a succession of acceptable moves (changes or stays). It possibly ends somewhere, in some desired state or, much before, in some trap. The (VR) approach starts with a precise definition of an acceptable move as a worthwhile move (change or stay) such that, at the individual or group level, motivation to move (desirability aspects of a move) is higher enough relative to resistance to move (feasibility aspects of this move), the various definitions of these terms being a major topic. A worthwhile move modelizes the degree of rationality of the agent, who can, each step, improve, improve enough (satisfy), locally optimize, optimize and improve with costs of being able to improve, $\cdots$. An acceptable transition is then modelized as a worthwhile transition, that is, a succession of worthwhile changes and stays. In this context, a trap is defined as an end of a worthwhile transition, worthwhile to reach, but not worthwhile to leave. The trap problem 
being to know when traps exist. An important instance of the trap problem refers to habituation processes (habit formation and breaking), where agents and organizations, progressively, perform more and more similar individual and collective actions in similar contexts, ending in habits and routines.

The behavioural part of our paper considers not only the existence, but also, the robustness of traps.

\subsection{The (VR) modelization of a worthwhile move}

The general formulation of a worthwhile move is given in Soubeyran $[49,50]$. It includes different variants. Here, to help the reader, we consider, first, one of its simplest formulations, and, then, present a specific formulation well adapted to the mathematical part of the paper.

\subsubsection{An example of a worthwhile individual move}

If, in the previous and current periods, an agent performs the bundles of activities $x \in X$ and $y \in X$, then, $x \curvearrowright y \neq x$ is a change, and $x \curvearrowright y=x$ is a stay. Each period, the main variational question is between choosing to stay, $x \curvearrowright y=x$ or to move, $x \curvearrowright y \neq x$, comparing scalar or vectorial advantages and inconveniences to change rather than to stay, $A(x, y) \in Y$ and $I(x, y) \in Y$, where $Y=\mathbf{R}$ or $Y=\mathbf{R}^{h}, h \geq 1$. These advantages and inconveniences generate both motivation and resistance to change rather than to stay $M=M(x, y)=U[A(x, y)] \in \mathbf{R}$ and $R=R(x, y)=D[I(x, y)] \in \mathbf{R}$, where $U[A] \in \mathbf{R}_{+}$and $D[I] \in \mathbf{R}_{+}$refer to positive and negative tensions (feelings), that is the utility and disutility of these advantages and inconveniences (more general formulations being given elsewhere). A move $x \curvearrowright y$ is worthwhile if motivation to change rather than to stay is high enough relative to resistance to change rather than to stay, that is, if $M(x, y) \geq \xi R(x, y)$, where $\xi>0$ defines the term 'high enough'. Let us see the simplest example of an advantage and an inconvenience to change rather than to stay.

An example of advantages to change rather to stay. Suppose that, in the current period, an agent, doing a bundle of activities $y \in X$, gets the scalar payoff (profit, utility) $g(y) \in \mathbf{R}$, while doing again the previous bundle of activities $x \in X$, gets the payoff $g(x) \in \mathbf{R}$. Then, his advantages to change rather than to stay are $A(x, y)=g(y)-g(x)$, if they are nonnegative. If $\bar{g}=\sup \{g(z), z \in X\}<+\infty$ represents the aspiration of this agent, then, $f(y)=\bar{g}-g(y) \geq 0$ refers to his residual unsatisfaction at $y$. This shows that $A(x, y)=g(y)-g(x)=[\bar{g}-f(y)]-[\bar{g}-f(x)]=-[f(y)-f(x)]$, that is $A(x, y)=-F(x, y)$, where $F(x, y)=f(y)-f(x) \geq 0$ refers to losses of changing, when they are nonnegative (unsatisfaction increases with changing).

An example of inconveniences to change rather than to stay. In a simple and scalar case, inconveniences to change rather than to stay, $I(x, y)=C(x, y)-C(x, x) \geq 0$, refer to the nonnegative difference between costs of being able to change, $C(x, y) \in \mathbf{R}_{+}$, and costs of being able to stay, $C(x, x) \in \mathbf{R}_{+}$. Usually $C(y, x) \neq C(x, y)$. If, furthermore, $C(x, x)=0$, and $C(x, y)>0$ when $y \neq x$, then, $q(x, y)=I(x, y)=C(x, y) \geq 0$ defines a quasi distance on $X$. See [47] for an explicit example. Notice that costs of being able to change $C(x, y)$, i.e. capability costs, must not be confused with traditional costs of doing, that is execution costs $\rho(y) \in \mathbf{R}_{+}$. These costs of doing $\rho(y)$ appear in the payoff $g(y)=r(y)-\rho(y)$, where $r(y) \in \mathbf{R}_{+}$are revenues.

Linear utility and disutility. In this simple example, as well as in the present paper, we will suppose that $U[A]=A$, and $D[I]=I$. Then, a move $x \curvearrowright y \neq x$ is worthwhile if $A(x, y) \geq \xi I(x, y)$, that is, if $g(y)-g(x) \geq \xi k(x) q(x, y)$.

\subsubsection{An example of a worthwhile collective move}

Consider a group of agents $J=\{1,2, \ldots, m\}$ which perform, in the current period, a collective activity $y=\left\{y_{1}, \cdots, y_{j}, \ldots, y_{m}\right\} \subset X$. Each agent $j$ of the group $J$ carries out the individual bundle of activities $y_{j} \in X$ and benefits from the individual payoff $g_{j}(y) \in \mathbf{R}$. The collective payoff of the group is the vector $g(y)=\left(g_{1}(y), \ldots, g_{j}(y), \ldots, g_{m}(y)\right) \in Y=\mathbf{R}^{J}$. Suppose that the goal 
of each agent is to improve his individual payoff. The ideal collective payoff of the group is $\bar{g}=$ $\left(\bar{g}_{1}, \ldots, \bar{g}_{j}, \ldots, \bar{g}_{m}\right)$, where $\bar{g}_{j}=\sup \left\{g_{j}(z), z \in X\right\}<+\infty$ is the agent $j$ aspiration and $f_{j}(y)=\bar{g}_{j}-$ $g_{j}(y) \geq 0$ is his residual need (unsatisfied aspiration), the collective vector of unsatisfactions being the vector $f(y)=\left(f_{1}(y), \ldots, f_{j}(y), \ldots, f_{m}(y)\right) \in Y$. Then, collective advantages to change rather than to stay are $A(x, y)=g(y)-g(x)=-F(x, y) \in K \subset Y$, while collective losses of changing rather than staying are $F(x, y)=f(y)-f(x) \in K$, where $K$ is a nonnegative cone.

Collective costs of being able to change rather than to stay are $C(x, y)=\left(C_{1}(x, y), \ldots, C_{j}(x, y), \ldots\right.$, $\left.C_{m}(x, y)\right) \in K$. Then, if they are nonnegative, collective inconveniences to change rather than to stay are $I(x, y)=C(x, y)-C(x, x) \in K$.

A collective worthwhile move is a worthwhile move for all members of the group, that is, $A(x, y) \geq_{K} \xi I(x, y)$.

In order to define individual capability costs $C_{j}(x, y) \in \mathbf{R}_{+}$for each member $j \in J$ of the group, the group can estimate a total capability cost $\mathbb{C}(x, y) \in \mathbf{R}_{+}$and shares it between its members, $C_{j}(x, y)=\theta_{j}(x) \mathbb{C}(x, y)$, where $\Sigma_{j \in J} \theta_{j}(x)=1, \theta_{j}(x)>0, j \in J$.

The total capability cost being a scalar quasi distance, $\mathbb{C}(x, y)=q(x, y) \in \mathbf{R}_{+}$, the vector of individual capability costs is

$C(x, y)=\theta(x) q(x, y)$, where the vector of shares is

$\theta(x)=\left(\theta_{1}(x), \ldots, \theta_{j}(x), \ldots, \theta_{m}(x)\right) \in K \backslash\{0\}$.

In this case inconveniences to change rather than to stay are $I(x, y)=\theta(x) q(x, y)$. Then, a collective move is worthwhile if $g_{j}(y)-g_{j}(x) \geq \xi \theta_{j}(x) q(x, y), \forall j \in J$, that is,

$A(x, y) \geq_{K} \xi \theta(x) q(x, y)$, i.e. $F(x, y)+\xi \theta(x) q(x, y) \leq_{K} 0$.

\subsubsection{An example of an individual worthwhile move with multiple objectives}

Consider, as before, an agent who performs a bundle of activities $y \in X$ in the current period, in order to satisfy several different objectives

$$
\left(g_{1}(y), \ldots, g_{j}(y), \ldots, g_{m}(y)\right)
$$

instead of a scalarized objective $g(y)=\Sigma_{j \in J} w_{j} g_{j}(y), w_{j}>0$ for $j \in J$. Then, the agent will have to practice mental accounting (Thaler [56]) to allocate the given total capability cost $\mathbb{C}(x, y) \in \mathbf{R}_{+}$ between his different activities to be able to compare each advantage to change rather than to stay $A_{j}(x, y)=g_{j}(y)-g_{j}(x)$ with the related inconvenience to change rather than to stay, $I_{j}(x, y)=$ $C_{j}(x, y)-C_{j}(x, x), j \in J$. Mental accounting allocates to each objective $j$ the capability cost $C_{j}(x, y)=$ $\theta_{j}(x) \mathbb{C}(x, y)$, where $, \Sigma_{j \in J} \theta_{j}(x)=1, \theta_{j}(x)>0, j \in J$. Then, as before, a worthwhile move is such that $g_{j}(y)-g_{j}(x) \geq \xi C_{j}(x, y), j \in J$.

\subsection{The (VR) modelization of a robust worthwhile move}

Let us show how the mathematical part of the present paper considers robust worthwhile moves, when vectorial advantages to change depend of an uncertain parameter $s \in S$ which belongs to a given subset $S$. In this way our set valued Ekeland equilibrium theorem gives a robust version of a vectorial equilibrium problem. This robust variational principle must be related to robust optimization programs $\min _{x \in X}\left\{f(x / s)\right.$, s.t $\left.F(x / s) \in K \subset \mathbf{R}^{\mathbf{m}}\right\}$, (Ben-Tal and Nemirovski [57]; Ben Tal et al. [51]), where $s \in S$ is the data element of the problem and $x$ is a decision variable. The data may be partly or fully 'uncertain', and all that is known about the data vector is that it belongs to a given uncertainty set $S$. Moreover the constraints $F(x / s) \in K$ must be satisfied, whatever the actual realization of $s \in S$.

\subsubsection{The presence of uncertain data s}

In this paper, at the level of a group, let us suppose that vectorial advantages to change rather than to stay $A(x, y, s)=(g(y / s)-h)-g(x / s)=f(x / s)-(f(y / s)+h)=-F(x, y, s)$, where $h>0$ 
modelizes the presence of a statu quo bias (see below), depend on some uncertain data $s \in S$ while vectorial inconveniences to change rather than to stay $\mathrm{I}(\mathrm{x}, \mathrm{y})$ do not. We also suppose that $I(x, y)=k q(x, y)$, where $k \in K \backslash\{0\}$. This means that shares of the total costs are held constant, that is $\theta(x)=k$. Then, a robust worthwhile change is such that $A(x, y, s) \geq_{K} \xi I(x, y)$ for all $s \in S$, where the worthwhile to change ratio $\xi=\psi(x)>0$ depends of the statu quo. In terms of losses of changing, we get $F(x, y, s)+k \psi(x) q(x, y) \leq_{K} 0$ for all $s \in S$.

Notice that the presence of uncertain data defines uncertain preferences which depend both on the known statu quo $x$ and the uncertain data $s \in S$, that is, $y \geq_{x, S} x$ iff $A(x, y, s) \geq_{K} \psi(x) I(x, y)$ for all $s \in S$, where $\xi=\psi(x)$. This situation differs from the case of variable preferences, when weights over the different objectives change, that is, when the cone $K=K(x)$ is changing with the statu quo (Bao et al. [44-46]).

\subsubsection{Hypothesis relative to $F$}

Let us write $F(x, y)=(F(x, y, s): s \in S)$, where $F(x, y, s)=-A(x, y, s)$ as above.

The subadditivity hypothesis. Eidelman and Crandall [58] defined the statu quo bias as an emotional bias, a preference for the current state of affairs, the status quo being taken as a reference point, and any change from it being perceived as a loss. When there are uncertain data $s \in S$, the variational rationality approach modelizes the status quo bias as follow. Advantages to change from the statu quo $x$ to $y$ include a statu quo bias $h>0$ when $A(x, y, s)=(g(y / s)-h)-g(x / s)$ if $y \neq x$ and $A(x, x, s)=0$ if $y=x$ for all $s \in S$. This shows that $A(x, z, s) \geq A(x, y, s)+A(y, z, s)$ for all $x, y, z \in X$, and all $s \in S$. Then,

(i) if $z \neq x$ and $y \neq x, y \neq z, A(x, z, s)=(g(z / s)-h)-g(x / s)=$ $[(g(y / s)-h)-g(x / s)]+[(g(z / s)-h)-g(y / s)]+h$.

This shows that $A(x, z, s)=A(x, y, s)+A(y, z, s)+h$, which implies that from $F(x, y, s)=$ $-A(x, y, s)$,

$F(x, z, s)=F(x, y, s)+F(y, z, s)-h$.

(ii) if $z \neq x$ and $y=x$ or $y=z, A(x, z, s)=A(x, y, s)+A(y, z, s)$. That is, $F(x, z, s)=F(x, y, s)+$ $F(y, z, s)$.

This shows that $F(x, z) \subset F(x, y)+F(y, z)-K$.

Other hypothesis on $F$. Their interpretations are the following.

(i) it exists $x_{0} \in X$ such that $F\left(x_{0}, x_{0}\right) \subset-K$ means that reference dependent vectorial advantages to stay $A(x, x, s) \geq 0$ are non negative for all $s \in S$.

(ii) it exists $\alpha \in \mathbf{R}$, it exists $s_{0} \in(-\infty,+\infty) k_{0}-K$ such that $F\left(x_{0}, X\right) \cap\left(-s_{0}+(-\infty,+\infty) k_{0}-\right.$ $K)=\emptyset$ tells us that losses $A\left(x_{0}, y, s\right)$ are bounded above in a given direction.

(iii) $F$ is left $\mathrm{K}-\mathrm{slm}$ in $(X, q)$ is a regularity hypothesis.

(iv) $\psi: X \rightarrow(0,+\infty)$ is such that $\psi\left(x^{\prime}\right) \geq \psi(x)$ if $F\left(x, x^{\prime}\right) \subset-K$, means that the worthwhile to change ratio $\xi=\psi(x)>0$ must increase when the agent or the group makes an improving change from $x$ to $x^{\prime}$, that is, when $A\left(x, x^{\prime}\right) \geq_{K} 0$. This hypothesis refers to the goal gradient hypothesis (Locke and Latham [59]; Kivetz et al. [60]), that is, as humans and other animals approach reaching a goal, their efforts towards that goal increase, because the more, each step, they want to improve.

For an explicit example of $F(x, y, s)$ where $F(x, y, s)=-A(x, y, s)$ and $A(x, y, s)=(g(y / s)-h)-$ $g(x / s)$, the reader can consider [47], where, in the dynamical context of the famous $O$ Ring theory of the limit of a firm, $g(x)=\varphi[Q(x), s(x)]-\rho(x)$ refers to the current profit of the firm, using a Cobb Douglas production function, the terminology coming from the NASA Apollo program. In the present paper, $g(x / s)=\varphi[Q(x), s(x), s]-\rho(x, s)$ generalizes $g(x)$, via the introduction of uncertain data ' $s$ ' (which have nothing to do with $s(x)$, which represents, in [47], the endogenous quality of the final good produced by the firm). 


\subsection{EVP principles as sufficient conditions for the existence of robust traps}

We are now in a good position to see the behavioural signification of our set valued Ekeland equilibrium Theorem 3.1. Given the behavioural interpretation of all the hypothesis listed in this theorem (see before), this result shows that, starting from any initial position $x_{0}$, there exists a robust $\operatorname{trap} \bar{x} \in X$ such that,

(a) it is worthwhile to directly move from $x_{0}$ to $\bar{x}$, for all $s \in S$, i.e. vectorial advantages to change rather than to stay, $A\left(x_{0}, \bar{x}, s\right)$, are high enough, more than $\psi\left(x_{0}\right)>0$, relative to vectorial inconveniences to change rather than to stay, $I\left(x_{0}, \bar{x}\right)=q\left(x_{0}, \bar{x}\right) k_{0}$ for all $s \in S$. That is, $A\left(x_{0}, \bar{x}, s\right) \geq_{K}$ $\psi\left(x_{0}\right) q\left(x_{0}, \bar{x}\right) k_{0}=\psi\left(x_{0}\right) I\left(x_{0}, \bar{x}\right)$, which is equivalent to, in term of set valued losses of changing, $F\left(x_{0}, \bar{x}\right)+\psi\left(x_{0}\right) q\left(x_{0}, \bar{x}\right) k_{0} \subset-K$.

(b) it is not worthwhile to change, starting from this robust trap $\bar{x}$, whatever is $s \in S$. That is,

$\forall x \in X \backslash\{\bar{x}\}, \exists s \in S$ such that $A(\bar{x}, x, s) \ngtr_{K} \psi(\bar{x}) q(\bar{x}, x) k_{0}$, or in terms of vectorial losses of changing,

$\forall x \in X \backslash\{\bar{x}\}, \exists s \in S$ such that $F(\bar{x}, x, s)+\psi(\bar{x}) q(\bar{x}, x) k_{0} \Varangle_{K} 0$.

The set valued formulation follows.

Then, our EVP principle shows in which circumstances traps exist and are robust to the unknown influences $s \in S$ of the external and internal environments of an agent or group of agents, like different possible experiences, contexts, physiological, physical, cognitive, emotional and social states, and reference points (including the statu quo, reservation values and aspirations) which lead to variable preferences. This is an important result in Behavioral Sciences, because it shows that, even in a world where so many things can change, an agent or a group of agents can be stucked in the middle, in a robust trap, being unable to reach his desires at the end. Then, variable preferences coming from the difficulty to predict the internal and external environment do not forbidd the existence of traps. As an application, this confirms, in a dynamic setting, the importance of the revolution offered by Kahneman and Tversky [61], Tversky and Kahneman [62], who emphasized the role of reference dependent preferences in static decision-making theory.

Remark: To save space, the interpretation of results given in Section 4 on equilibrium problems, vector optimization and fixed point theorems in term of robust desired ends (robust desires) will be given elsewhere, using the (VR) variational rationality approach of human dynamics $[49,50]$.

\section{Acknowledgements}

The authors are grateful to the reviewers and the editors for their valuable comments and suggestions, which greatly improved the paper.

\section{Disclosure statement}

No potential conflict of interest was reported by the authors.

\section{Funding}

The first two authors were supported by the National Natural Science Foundation of China [grant number 11471236], [grant number 11561049].

\section{References}

[1] Ekeland I. On the variational principle. J Math Anal Appl. 1974;47:324-353.

[2] Ekeland I. Nonconvex minimization problems. Bull Amer Math Soc. 1979;1:443-474.

[3] Göpfert A, Riahi H, Tammer C, Zălinescu C. Variational methods in partially ordered spaces. New York (NY): Springer-Verlag; 2003.

[4] Chen GY, Huang XX, Yang XG. Vector optimization: set-valued and variational analysis. Berlin: Springer-Verlag; 2005. 
[5] Bao TQ, Mordukhovich BS. Relative Pareto minimizers for multiobjective problems: existence and optimality conditions. Math Program. 2010;122:301-347.

[6] Bednarczuk EM, Zagrodny D. Vector variational principle. Arch Math (Basel). 2009;93:577-586.

[7] Dentcheva D, Helbig S. On variational principles, level sets, well-posedness, and $\epsilon$-solutions in vector optimization. J Optim Theory Appl. 1996;89:325-349.

[8] Finet C, Quarta L, Troestler C. Vector-valued variational principles. Nonlinear Anal. 2003;52:197-218.

[9] Flores-Bazán F, Gutiérrez C, Novo V. A Brézis-Browder principle on partially ordered spaces and related ordering theorems. J Math Anal Appl. 2011;375:245-260.

[10] Göpfert A, Tammer C, Zalinescu C. On the vectorial Ekeland's variational principle and minimal point theorems in product spaces. Nonlinear Anal. 2000;39:909-922.

[11] Gutiérrez C, Jiménez B, Novo V. A set-valued Ekeland's variational principle in vector optimization. SIAM J Control Optim. 2008;47:883-903.

[12] Ha TXD. Some variants of the Ekeland variational principle for a set-valued map. J Optim Theory Appl. 2005;124:187-206.

[13] Hamel AH. Equivalents to Ekeland's variational principle in uniform spaces. Nonlinear Anal. 2005;62:913-924.

[14] Khanh PQ, Quy DN. Versions of Ekeland's variational principle involving set perturbations. J Glob Optim. 2013;57:951-968.

[15] Liu CG, Ng KF. Ekeland's variational principle for set-valued functions. SIAM J Optim. 2011;21:41-56.

[16] Németh AB. A nonconvex vector minimization problem. Nonlinear Anal. 1986;10:669-678.

[17] Qiu JH. A generalized Ekeland vector variational principle and its applications in optimization. Nonlinear Anal. 2009;71:4705-4717.

[18] Qiu JH. On Ha's version of set-valued Ekeland's variational principle. Acta Math Sinica Engl Ser. 2012;28:717-726.

[19] Qiu JH. Set-valued quasi-metrics and a general Ekeland's variational principle in vector optimization. SIAM J Control Optim. 2013;51:1350-1371.

[20] Qiu JH. A pre-order principle and set-valued Ekeland variational principle. J Math Anal Appl. 2014;419:904-937.

[21] Qiu JH, He F. A general vectorial Ekeland's variational principle with a p-distance. Acta Math Sinica Engl Ser. 2013;29:1655-1678.

[22] Qiu JH, Li B, He F. Vector Ekeland's variational principle with a w-distance and its equivalent theorems. Acta Math Sci. 2012;32:2221-2236.

[23] Tammer C. A generalization of Ekeland's variational principle. Optimization. 1992;25:129-141.

[24] Tammer C, Zălinescu C. Vector variational principle for set-valued functions. Optimization. 2011;60:839-857.

[25] Oettli W, Théra M. Equivalents of Ekeland's principle. Bull Austral Math Soc. 1993;48:385-392.

[26] Blum E, Oettli W. From optimization and variational inequalities to equilibrium problems. Math Stud. 1994;63:123-145.

[27] Al-Homidan S, Ansari QH, Yao JC. Some generalizations of Ekeland-type variational principle with applications to equilibrium problems and fixed point theory. Nonlinear Anal. 2008;69:126-139.

[28] Alleche B, Rădulescu VD. The Ekeland variational principle for equilibrium problems revisited and applications. Nonlinear Anal Real World Appl. 2015;23:17-25.

[29] Bianchi M, Kassay G, Pini R. Existence of equilibria via Ekeland's principle. J Math Anal Appl. 2005;305:502-512.

[30] Bianchi M, Kassay G, Pini R. Ekeland's principle for vector equilibrium problems. Nonlinear Anal. 2007;66:14541464.

[31] Farkas C, Molnár AE. A generalized variational principle and its application to equilibrium problems. J Optim Theory Appl. 2013;156:213-231.

[32] Gong X. Ekeland's principle for set-valued vector equilibrium problems. Acta Math Sci. 2014;34B:1179-1192.

[33] Hamel AH. Equivalents to Ekeland's variational principle in uniform spaces. Nonlinear Anal. 2005;62:913-924.

[34] Lin LJ, Du WS. Ekeland's variational principle, minimax theorems and existence of nonconvex equilibria in complete metric spaces. J Math Anal Appl. 2006;323:360-370.

[35] Qiu JH. An equilibrium version of vectorial Ekeland variational principle and its applications to equilibrium problems. Nonlinear Anal Real World Appl. 2016;27:26-42.

[36] Qiu JH. An equilibrium version of set-valued Ekeland variational principle and its applications to set-valued vector equilibrium problems. Acta Math Sinica Engl Ser. 2017;33:210-234.

[37] Zeng J, Li SJ. An Ekeland's variational principle for set-valued mappings with applications. J Comput Appl Math. 2009;230:477-484.

[38] Alleche B, Rădulescu VD. Set-valued equilibrium problems with applications to Browder variational inclusions and to fixed point theory. Nonlinear Anal Real World Appl. 2016;28:251-268.

[39] László S, Viorel A. Densely defined equilibrium problems. J Optim Theory Appl. 2015;166:52-75.

[40] Kristály A, Varga C. Set-valued versions of Ky Fan's inequality with application to variational inclusion theory. J Math Anal Appl. 2003;282:8-20.

[41] Cobzas S. completeness in quasi-metric spaces and Ekeland variational principle. Topol Appl. 2011;158:10731084. 
[42] Cobzas S. Functional analysis in asymmetric normed spaces. Frontiers in mathematics. Basel: Birkhäuser/Springer Basel AG; 2013.

[43] Karapinar E, Romaguera S. On the weak form of Ekeland's variational principle in quasi-metric spaces. Topol Appl. 2015;184:54-60.

[44] Bao TQ, Mordukhovich BS, Soubeyran A. Variational analysis in psychological modeling. J Optim Theory Appl. 2015;164:290-315.

[45] Bao TQ, Mordukhovich BS, Soubeyran A. Fixed points and variational principles with applications to capability theory of wellbeing via variational rationality. Set-Valued Var Anal. 2015;23:375-398.

[46] Bao TQ, Mordukhovich BS, Soubeyran A. Minimal points, variational principles, and variable preferences in set optimization. J Nonlinear Convex Anal. 2015;16:1511-1537.

[47] Bao TQ, Khanh PQ, Soubeyran A. Variational principles with generalized distances and the modelization of organizational change. Optimization. 2016;65:2049-2066.

[48] Bao TQ, Théra MA. On extended versions of Dancs-Hegedus-Medvegyev's fixed point theorem. Optimization. 2017;66:875-887.

[49] Soubeyran A. Variational rationality, a theory of individual stability and change: worthwhile and ambidextry behaviors, GREQAM, Aix-Marseillle University. Preprint 2009.

[50] Soubeyran A. Variational rationality and the "unsatisfied man": routines and course pursuit between aspirations, capabilities and beliefs, GREQAM, Aix-Marseillle University. Preprint 2010.

[51] Ben-Tal A, El Ghaoui L, Nemirovski A. Robust optimization. Princeton (NJ): Princeton University Press; 2009.

[52] Gerstewitz (Tammer) Chr. Nichtkonvexe Dualität in der Vektoroptimierung. Wiss Zeitschr TH LeunaMerseburg. 1983;25:357-364.

[53] Adan M, Novo V. Proper efficiency in vector optimization on real linear spaces. J Optim Theory Appl. 2004;121:515-540.

[54] Kelly JC. Bitopological spaces. Proc London Math Soc. 1963;13:71-89.

[55] Köthe G. Topological vector spaces I. Berlin: Springer-Verlag; 1969.

[56] Thaler RH. Mental accounting matters. J Behav Decis Making. 1999;12(3):183-206.

[57] Ben-Tal A, Nemirovski A. Robust convex optimization. Math Oper Res. 1998;23(4):769-805.

[58] Eidelman S, Crandall CS. Bias in favor of the status quo. Soc Personality Psychol Compass. 2012;6(3):270-281.

[59] Locke EA, Latham GP. Goal setting, a motivational technique that works. Englewood Cliffs (NJ): Prentice Hall; 1984

[60] Kivetz R, Urminsky O, Zheng Y. The goal-gradient hypothesis resurrected: purchase acceleration, illusionary goal progress, and customer retention. J Marketing Res. 2006;43(1):39-58.

[61] Kahneman D, Tversky A. Prospect theory: an analysis of decision under risk. Econometrica. 1979;47(2):263-291.

[62] Tversky A, Kahneman D. Loss aversion in riskless choice: a reference-dependent model. Q J Econ. 1991;106(4):1039-1061. 